\title{
The Combinatorics of Cache Misses during Matrix Multiplication ${ }^{1}$
}

\author{
Philip J. Hanlon \\ Department of Mathematics, University of Michigan, Ann Arbor, Michigan 48109
}

\section{Dean Chung}

Amazon.com, Inc., Seattle, Washington

\section{Siddhartha Chatterjee and Daniela Genius}

Department of Computer Science, University of North Carolina, Chapel Hill, North Carolina 27599

\author{
Alvin R. Lebeck
}

Department of Computer Science, Duke University, Durham, North Carolina 27706

and

\section{Erin Parker}

Department of Computer Science, University of North Carolina, Chapel Hill, North Carolina 27514

Received March 17, 2000; revised September 1, 2000

In this paper we construct an analytic model of cache misses during matrix multiplication. The analysis in this paper applies to square matrices of size $2^{m}$ where the array layout function is given in terms of a function $\Theta$ that interleaves the bits in the binary expansions of the row and column indices. We first analyze the number of cache misses for direct-mapped caches and then indicate how to extend this analysis to $\mathbb{A}$-way associative caches. The work in this paper accomplishes two things. First, we construct fast algorithms to estimate the number of cache misses. Second, we develop a theoretical understanding of cache misses that will allow us, in subsequent work, to approach the problem of minimizing cache misses by appropriately choosing the bit interleaving function that goes into the array layout function. $\quad$ (C) 2001 Academic Press

${ }^{1}$ This work was supported in part by DARPA Grant DABT63-98-1-0001, NSF Grants EIA-97-26370 and CDA-95-12356, NSF Career Award MIP-97-02547, The University of North Carolina at Chapel Hill, Duke University, and an equipment donation through Intel Corporation's Technology for Education 2000 Program. The views and conclusions contained herein are those of the authors and should not be interpreted as representing the official policies or endorsements, either expressed or implied, of DARPA or the U.S. Government. 


\section{INTRODUCTION}

As the gap between processor cycle time and main memory access time continues to widen, effective use of the memory hierarchy becomes ever more critical to overall program performance. Caches can help alleviate the CPU-memory gap by satisfying most memory references at close to processor speed ( 1 to 3 cycles). Unfortunately, programs that do not exhibit good memory reference locality cannot exploit the potential benefits of caches.

For scientific computations that repeatedly access large data sets, good locality of reference is essential at the algorithm level for high performance. Such locality can either be temporal, in which a single data item is reused repeatedly, or spatial, in which a group of data items "adjacent" in space are used in temporal proximity. High-performance dense linear algebra codes rely on good spatial and temporal locality of reference for their performance. In this paper, we focus on an analysis of matrix multiplication, the workhorse of modern linear algebraic algorithms.

Our previous studies demonstrated an intimate relationship between the layout of the arrays in memory and the performance of the routine [1,2]. This early work experimentally showed the benefits of using array layout functions based on interleaving the bits in the binary expansions of the row and column indices of arrays. This paper complements our earlier empirical studies by providing an analytical framework for analyzing the cache behavior of matrix multiplication in the presence of such array layout functions. Future work will use this framework in an optimization context, to determine array layouts that minimize the number of cache misses. The techniques applied in the analysis presented in this paper are specific to the matrix multiplication example, and may not generalize easily to other programs.

The remainder of this section provides the background of the cache analysis problem. Section 1.1 provides a brief overview of cache memory basics. Section 1.2 describes our analysis framework - both the similarities to earlier work and the critical differences that require us to use completely different techniques. Section 1.3 discusses array layout functions based on bit interleaving. Section 1.4 reiterates the goals of our analysis and provides a roadmap of the remainder of the paper.

\subsection{Basics of Cache Memory}

We assume a simplified memory hierarchy that processes one memory access at a time, with no distinction between memory reads and writes.

The structure of a single level of a memory hierarchy-called a cache-is generally characterized by three parameters: Associativity, Block size, and Capacity. Capacity and block size are in units of the minimum memory access size (usually one byte). A cache can hold a maximum of $C$ bytes. However, due to physical constraints, the cache is divided into cache frames of size $B$ that contain $B$ contiguous bytes of memory - called a memory block. The associativity $A$ specifies the number of different frames in which a memory block can reside; such a set of $A$ cache frames is also called a cache set. If a block can reside in any frame (i.e., $A=\frac{C}{B}$ ), the cache is said to be fully associative; if $A=1$, the cache is direct-mapped; otherwise, the cache is $A$-way set associative. 
For a given memory access, the hardware inspects the cache to determine if the corresponding memory element is resident in the cache. This is accomplished by using an indexing function to locate the appropriate cache set that may contain the memory block. If the memory block is resident, a cache hit is said to occur, and the cache satisfies the access after its access latency. If the memory block is not resident, a cache miss is said to occur. In this case, the memory block is fetched from a lower level of the memory hierarchy. If all $A$ frames in the cache set contain valid data, then one of them must be freed to accommodate the current memory block. This is a nontrivial decision for any cache organization other than direct-mapped. The (fast on-line) algorithm used to determine the block to evict from the cache set is called the replacement policy of the cache. A commonly used replacement policy is Least Recently Used (LRU), in which the memory block that was last accessed furthest in the past is chosen for replacement. We will analyze this replacement policy in Section 5.

From an architectural standpoint, cache misses fall into one of three classes [7]. Note that each class of miss can occur in any cache that is not fully associative.

- A compulsory miss is one that is caused by referencing a previously unreferenced memory block. Eliminating a compulsory miss requires prefetching the data, either by an explicit prefetch operation or by placing more data items in a single memory block.

- A reference that is not a compulsory miss but misses in a fully associative cache with LRU replacement is classified as a capacity miss. Capacity misses are caused by referencing more memory blocks than can fit in the cache. Restructuring the program to re-use blocks while they are in cache can reduce capacity misses.

- A reference that hits in a fully associative cache but misses in an $A$-way setassociative cache is classified as a conflict miss (or interference miss). A conflict miss to block $\mathrm{X}$ indicates that block $\mathrm{X}$ has been referenced in the recent past, since it is contained in the fully associative cache, but at least $A$ other memory blocks that map to the same cache set have been accessed since the last reference to block X. Eliminating conflict misses requires transforming the program to change either the memory allocation and/or layout of the two arrays (so that contemporaneous accesses do not compete for the same sets) or the manner in which the arrays are accessed. At the program source level, interference misses can be further subdivided based on whether the interfering blocks come from different parts of a single array, or from different arrays. The miss is called a self-interference miss in the former case and a cross-interference miss in the latter case [8].

\subsection{An Analysis Framework}

Our general model for counting cache misses follows the framework used in previous work [5], with one significant difference. We first explain the common framework, then highlight the key difference in our version of the problem that necessitates entirely new solution techniques.

Consider the following loop nest for matrix multiplication (the so-called $i k j$ variant), which will be the specific computation whose cache behavior we analyze in the remainder of this paper. 


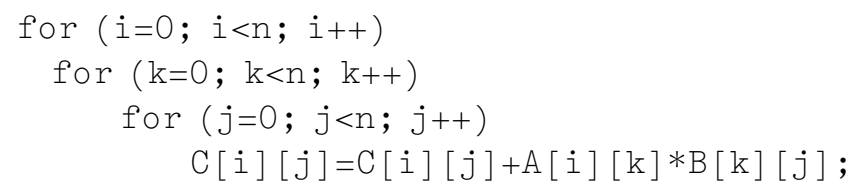

An array $A$ has an associated layout function $\mathscr{L}_{A}$, which is a 1-1 map from $[0, n-1] \times[0, n-1]$ to the memory address space $\mathbb{Z}_{0}^{+}$. Applying this map to an element of an array produces the byte address of that array element.

We assume a two-level memory hierarchy (i.e., a cache backed by a conceptually infinite main memory), with a direct-mapped cache with block size of $B$ bytes and total capacity of $C$ bytes (and therefore $p=C / B$ sets). The quantities $B$ and $C$ are always powers of two for technological reasons, so we will assume that $p=2^{\rho}$. We also assume that main memory is large enough to hold all the data referenced by the program. The function $\mathscr{B}$ converts a memory byte address into a memory block address (with $\mathscr{B}(a)=\lfloor a / B\rfloor$ ). The function $\mathscr{S}$ converts a memory block address to the cache set to which it maps (thus, $\mathscr{S}(b)=b \bmod p$ ).

In the remainder of the paper we will work in units of array elements rather than bytes. Given that 32 bytes is a popular block size for first-level caches in many modern machines, and that double-precision numbers are represented with eight bytes, we will assume in this paper that memory blocks and cache blocks hold four array elements.

The goal of cache analysis is to efficiently estimate the number of capacity and conflict misses of a given code fragment, given the numerical value of the loop bounds, a cache configuration, and the layout functions of the arrays. For example, in the matrix multiplication example, to formulate the conditions under which the reference $A_{i, k}$ misses at iteration point $(u, v, w)$ because it was replaced by reference $B_{k, j}$, let $\left(u^{\prime}, v^{\prime}, w^{\prime}\right)=\operatorname{Last}(u, v, w)$ be the most recent iteration point that accessed $b_{A}=\mathscr{B}\left(\mathscr{L}_{A}(u, w)\right)$, the block being accessed by the reference $A_{i, k}$ at iteration point $(u, v, w)$. Let $(x, y, z)$ be an iteration point between $\left(u^{\prime}, v^{\prime}, w^{\prime}\right)$ and $(u, v, w)$ in lexicographic order at which the memory block $b_{B}=\mathscr{B}\left(\mathscr{L}_{B}(z, y)\right)$ accessed by reference $B_{k, j}$ displaced block $b_{A}$ from cache. This condition is satisfied iff

$$
\mathscr{S}\left(\mathscr{B}\left(\mathscr{L}_{A}(u, w)\right)\right)=\mathscr{S}\left(\mathscr{B}\left(\mathscr{L}_{B}(z, y)\right)\right)
$$

Equation (1) captures both capacity and conflict misses but does not distinguish between the two. (Discriminating between these miss classes would require the additional ability to ascertain the hit/miss status of the reference in a fully associative cache.) It does not capture compulsory misses, as such misses correspond to iterations $w$ for which Last is not defined. We use the term replacement misses to encompass capacity and conflict misses. We omit compulsory misses from the scope of this paper for two reasons: they are unavoidable misses that cannot be reduced by optimization techniques, and they need to be formulated completely differently.

It is clear that a simple strategy to count misses is through simulation of the code. This is exactly what cache simulators do. The main drawback of simulation is its slowness: it takes time proportional to the actual execution of the code, 
usually with a significant multiplicative factor (10-100 is typical). In our matrix multiplication example, this time is $\Theta\left(n^{3}\right)$. Our interest is in much faster algorithms that work by exploiting the combinatorial structure of misses rather than by simulation, and whose existence is suggested by the regularity of the array access patterns and the limited number of cache sets to which they map. We will in fact demonstrate algorithms that accurately compute the number of cache misses for the matrix multiplication example in $O(\max (\log n, \log (C / B)))$ time.

Previous work [5] at this point introduces two additional constraints to make the problem tractable. First, it assumes that the layout functions are row or column-major, which is affine in the array coordinates. We will subsequently use the term canonical layout to refer to these two layout functions. Second, it assumes that Last can be obtained through reuse vectors, which occurs when the array index expressions are uniformly generated in addition to being affine in the LCVs. These two conditions keep everything within the polyhedral model [3], which has been well-studied and for which counting algorithms are well-known [9]. It is at this point that our work diverges from previous work.

Prior empirical evidence $[1,2,4]$ suggests that alternative array layout functions such as Morton order [2] provide better cache behavior than canonical layout functions for many dense linear algebra codes. Such layout functions are described in terms of interleavings of the bits in the binary expansions of the array coordinates rather than as affine functions of the numerical values of these quantities. This single change puts our version of the problem beyond the scope of the solution techniques for the polyhedral model. We will therefore need to investigate different techniques for counting the number of solutions to equations such as Eq. (1).

\subsection{Array Layouts Based on Bit Interleaving}

In developing this model of alternative array layouts, we assume that $n=2^{m}$, so that the bit representation of an array index will have $m$ bits, with the least significant bit (LSB) numbered 0 and the most significant bit (MSB) numbered $m-1$. We identify the binary sequence $s_{m-1} \cdots s_{0}$ with the nonnegative integer $s=$ $\sum_{i=0}^{m-1} s_{i} 2^{i}$. We denote by $B_{m}$ the set of all binary sequences of length $m$, and extend the above identification to identify $B_{m}$ with interval $\left[0,2^{m}-1\right]$.

We will describe a family of nonlinear layout functions parameterized by a single parameter $\sigma$, as follows. An $(m, m)$-interleaving, $\sigma$, is a $2 m$-bit binary sequence containing $m 0 \mathrm{~s}$ and $m 1 \mathrm{s.}^{2}$ It describes the order in which bits from the two array coordinates are interleaved to linearize the array in memory. Given $\sigma$, define its characteristic sequence $\chi_{\sigma}$ to be the sequence with entries $f_{i}$ and $s_{i}$ defined by replacing the $(i+1)$ st 0 from the right in $\sigma$ by $f_{i}$ and the $(i+1)$ st 1 from the right in $\sigma$ by $s_{i}$. (The letters $f$ and $s$ are chosen for mnemonic reasons: they are the initial letters of the words "first" and "second.")

Example 1 . Let $m=4$ and let $\sigma=10110010$. Then $\chi_{\sigma}=s_{3} f_{3} s_{2} s_{1} f_{2} f_{1} s_{0} f_{0}$. Next, let $m=3$ and let $\sigma=010011$. In this case, $\chi_{\sigma}=f_{2} s_{2} f_{1} f_{0} s_{1} s_{0}$.

\footnotetext{
${ }^{2}$ We could generalize this notation to $(m, n)$-interleavings for $m \neq n$. We choose not to do so as we do not need such generality for this paper.
} 
Given an $(m, m)$-interleaving $\sigma$, define a map

$$
\Theta: B_{m} \times B_{m} \rightarrow B_{2 m}
$$

in the following way. If $a=a_{m-1} \cdots a_{1} a_{0} \in B_{m}$ and $b=b_{m-1} \cdots b_{1} b_{0} \in B_{m}$, then $\Theta(a, b)$ is the sequence obtained by replacing each $f_{i}$ in $\chi_{\sigma}$ by $a_{i}$ and each $s_{i}$ in $\chi_{\sigma}$ by $b_{i}$. We extend this notation to consider $\Theta$ as a map from $\left[0,2^{m}-1\right] \times$ $\left[0,2^{m}-1\right] \rightarrow\left[0,2^{2 m}-1\right]$ by identifying nonnegative integers and their binary expansions. We call $\Theta$ the mixing function indexed by $\sigma$. Note that $\Theta(0,0)=0$ for any $\sigma$.

ExAmPLE 2. Let $m=4$ and let $\sigma=01101001$ so that $\chi_{\sigma}=f_{3} s_{3} s_{2} f_{2} s_{1} f_{1} f_{0} s_{0}$. Then

$$
\Theta(12,5)=\Theta(1100,0101)=10110001=128+32+16+1=177 .
$$

Next, let $\sigma=10110010$ so that $\chi_{\sigma}=s_{3} f_{3} s_{2} s_{1} f_{2} f_{1} s_{0} f_{0}$. In this case,

$$
\Theta(9,6)=\Theta(1001,0110)=01110001=64+32+16+1=113 .
$$

Many popular layout functions fall into this class. For example, row-major layout corresponds to the signature $\sigma=\overbrace{0 \ldots 0}^{n} \overbrace{1 \cdots 1}^{n}$; column-major layout corresponds to the signature $\sigma=\overbrace{1 \cdots 10 \cdots 0}^{n} \overbrace{10}^{n}$; pure Morton layout corresponds to the signature $\sigma=\overbrace{01 \cdots 01}^{n}$; a combination of Morton layout with $2^{k} \times 2^{k}$ tiles arranged in row-major order corresponds to the signature $\sigma=\overbrace{01 \cdots 01}^{2(n-k)} \overbrace{0 \cdots 0}^{k} \overbrace{1 \cdots 1}^{k}$; and so on.

We are now ready to discuss the matter of the layout functions of the three arrays in our matrix multiplication example. Given an arbitrary array element indexed $(r, c)$, the quantity $\Theta(r, c)$ gives the position of the element $(r, c)$ relative to the starting position of the array in memory. We use the generic notation $\mu$ to denote this starting address. Specifically, we assume the following forms of layout functions for $A, B$, and $C$ :

$$
\begin{aligned}
& \mathscr{L}_{A}(r, c)=\mu_{1}+\Theta(r, c) \\
& \mathscr{L}_{B}(r, c)=\mu_{2}+\Theta(r, c) \\
& \mathscr{L}_{C}(r, c)=\mu_{3}+\Theta(r, c) .
\end{aligned}
$$

\subsection{Goals and Structure of the Paper}

Our overall goal, to be studied in a subsequent paper, is to find the layout functions of the form shown above that minimize cache misses. In this paper, we create 
an analytic model of cache misses using layout functions of this form, and we use this model to estimate the number of cache misses in the matrix multiplication example. These results will form the basis for the analysis in future work. Our model in this paper is based on three important assumptions that limit the scope of the analysis. First, we assume that the matrices being multiplied are square and have nonoverlapping memory images. Second, we assume that a memory block holds four matrix elements. Third, for most of the paper, we assume a direct-mapped cache.

The counting of cache misses for the matrix multiplication example is, in the end, a giant case analysis of all possible patterns of interference among the various arrays. Fortunately, this analysis ultimately reduces to solving two enumeration problems, which are then adapted and augmented in diverse ways, and finally combined using inclusion-exclusion. We first discuss the two enumeration problems and their solutions in an abstract setting in Section 2. We then adapt these algorithms to the cache model in Section 3 and to the problem of counting cache misses in Section 4. We extend our analysis to set-associative caches in Section 5 and conclude in Section 6.

\section{TWO ENUMERATION PROBLEMS}

In this section, we study a pair of counting problems which together form the foundation for our enumeration of cache misses. We will not attempt to determine closed-form expressions for these numbers - almost certainly the answers to these questions cannot be put in elegant closed forms. Instead, our goal will be to describe efficient algorithms to determine the number of solutions.

We will let $n=2^{m}$ and $p=2^{\rho}$ be as in the last section. For any positive integer $q$ we will let $B_{q}$ denote the number of binary sequences $e=e_{q-1} \cdots e_{1} e_{0}$ of length $q$. When convenient, we will treat $e$ as a nonnegative integer in the range 0 to $2^{q}-1$ using the usual notion of binary representation.

In this section, we develop methods to count solutions $(a, b, c)$ to certain kinds of equations modulo $2^{\rho}$. Our analysis proceeds by examining constraints on the $i$ th bits on both sides of the equation for $0 \leqslant i \leqslant 2 m-1$. Since we are counting solutions modulo $2^{\rho}$, we can disregard any conditions on bits $\rho, \rho+1, \ldots, 2 m-1$ in the case that $2 m>\rho$. Therefore, any bits of $a, b, c$ which are unconstrained by our analysis of the first $\rho$ bits in our equation can be chosen arbitrarily, each contributing a factor of 2 to the number of solutions.

By this reasoning, it is straightforward to extend these methods from the case $2 m \leqslant \rho$ to the case $2 m>\rho$. Therefore, to simplify the exposition, we assume that $2 m \leqslant \rho$ for the remainder of this section.

\subsection{Algorithm $\mathbf{A B}(d)$}

Given an $(m, m)$-interleaving $\sigma$, an integer $d$ with a $\rho$-bit binary expansion, and an initial carry $k_{0} \in\{0,1\}$, we want to determine $\mathbf{A B}(\mathbf{d})$, the number of triples $(a, b, c) \in B_{m}^{3}$ such that

$$
\Theta(a, b)=\Theta(b, c)+d+k_{0} \bmod 2^{\rho}
$$


under the condition that $2 m \leqslant \rho$. A correct but inefficient algorithm would enumerate all possible triples $(a, b, c)$ and check satisfiability of Eq. (2) for each triple. Such an algorithm would have time complexity of $O\left(8^{m}+\rho\right)$. The basic technique that we will use to derive an efficient algorithm of time complexity $O(\max (m, \rho))$ is to reason about individual bits of the terms on either side of the equation in terms of whether they propagate or generate carry bits. We will denote by $k_{i}$ the carry input at bit position $i$ (or, equivalently, the carry output at position $i-1$ ). Note that $k_{0}$, the carry input at the least significant bit, is supplied.

The first observation is that we can simplify the problem based on the values of bits $d_{\rho-1}$ through $d_{2 m}$.

Definition 2.1 (Consistency of $d$ ). Let $\sigma$ be an $(m, m)$-interleaving and let $d=d_{\rho-1} \cdots d_{0} \in B_{\rho}$. Let $r=[u, \ldots, v]$ be a subsequence of $P=[0, \ldots, \rho-1]$. We say that $d$ is $\epsilon$-consistent on $r$ if $d_{j}=\epsilon$ for all $j \in r$. We say that $d$ is inconsistent on $r$ if it is neither 0 -consistent not 1 -consistent on $r$.

LEMMA 2.1. Equation (2) has no solutions if $d$ is inconsistent on $[2 m, \ldots, \rho-1]$. For $\epsilon \in\{0,1\}$, if $d$ is $\epsilon$-consistent on $[2 m, \ldots, \rho-1]$, then Eq. (2) has solutions iff $k_{2 m}=k_{\rho}=\epsilon$.

Proof. By case analysis on bits $d_{\rho-1}$ through $d_{2 m}$.

This reduces the original problem to that of counting the number of solutions to a reduced system $E$ of $2 m$ bit-equations, and separating the solutions of $E$ based on the value of $k_{2 m}$ that they produce. Let $n_{\epsilon}$ be the number of solutions of $E$ that produce $k_{2 m}=\epsilon$, for $\epsilon \in\{0,1\}$. Then we have the following expression for $\mathbf{A B}(\mathbf{d})$ :

$$
\mathbf{A B}(\mathbf{d})= \begin{cases}n_{0}, & \text { if } \quad d_{\rho-1}=\cdots=d_{2 m}=0 \\ n_{1} & \text { if } d_{\rho-1}=\cdots=d_{2 m}=1 \\ 0, & \text { otherwise. }\end{cases}
$$

We will now give an algorithm to determine the pair $\left(n_{0}, n_{1}\right)$.

Let us label the $2 m$ components of $E$ with the numbers 0 through $2 m-1$, with $t$ being the label of the equation corresponding to bit position $t$. Bit equation $t$ has one of two forms,

$$
\begin{aligned}
& b_{i}=c_{i}+d_{t} \\
& a_{i}=b_{i}+d_{t},
\end{aligned}
$$

where $0 \leqslant i<m$. For any fixed $i$, there is exactly one equation of form (4) and one equation of form (5) (of course, with different values of $t$ ). Of these, call the equation with larger value of $t$ the major $i$-equation, an the equation with smaller value of $t$ the minor $i$-equation.

The + in the above equations is to be interpreted as binary addition, with hidden carry bits. To make this explicit, we rewrite the component equations in a 
more elaborate form, using the operations exclusive-or (denoted $\oplus$ ) and majority (denoted MAJ). For Eq. (4) we get

$$
\begin{gathered}
b_{i}=c_{i} \oplus d_{t} \oplus k_{t} \\
k_{t+1}=\operatorname{MAJ}\left(c_{i}, d_{t}, k_{t}\right)
\end{gathered}
$$

while for Eq. (5) we get

$$
\begin{gathered}
a_{i}=b_{i} \oplus d_{t} \oplus k_{t} \\
k_{t+1}=\operatorname{MAJ}\left(b_{i}, d_{t}, k_{t}\right) .
\end{gathered}
$$

Our interest is not so much in specific values of the bits $a_{i}, b_{i}$, and $c_{i}$, but rather on the terminal carry $k_{2 m}$ that any particular assignment of bits produces. As $b$ is the only variable that occurs on both sides of component equations, a particular choice of $b$ uniquely determines values of $a$ and $c$. We will therefore use the bits of $b$ to collect solution triples that generate a common terminal carry. Looking at the behavior of component equation $t$ for a specific choice of $b_{i}$, we observe that it has three possible modes.

1. $k_{t+1}=k_{t}$. We call this mode Propagate, or $P$ for short.

2. $k_{t+1}=0$, independent of $k_{t}$. We call this mode 0-Generate, or $G_{0}$ for short.

3. $k_{t+1}=1$, independent of $k_{t}$. We call this mode 1-Generate, or $G_{1}$ for short.

The following lemma relates these modes to the choice of value $b_{i}$.

Lemma 2.2. Equation (4) behaves in mode $P$ if we set $b_{i}=d_{t}$ and in mode $G_{d_{t}}$ if $b_{i}=\bar{d}_{t}$. Equation (5) behaves in mode $G_{d_{t}}$ if we set $b_{i}=d_{t}$ and in mode $P$ if $b_{i}=\bar{d}_{t}$.

Proof. Simple case analysis based on possible values of $b_{i}$ and of $k_{t}$.

The key idea in the algorithm is to capitalize on the $G_{0}$ and $G_{1}$ modes. Consider $b_{m-1}$, the most significant bit of $b$. The major $(m-1)$-equation occurs at position $2 m-1$, and the minor $(m-1)$-equation occurs at some position $s$ with $s<2 m-1$. Depending on the form of equation $2 m-1$ and the value of $d_{2 m-1}$, one of the two choices for $b_{m-1}$ will lead to a $G_{\epsilon}$-mode (with $\epsilon \in\{0,1\}$ ). This means that no matter what values we assign to bits $b_{m-2}$ through $b_{0}$, they will all contribute to $n_{\epsilon}$. We can therefore increment $n_{\epsilon}$ by $2^{m-1}$. The other choice of $b_{m-1}$ will lead to $P$-mode for the major equation (and some mode for the minor equation as determined by Lemma 2.2). In this case, we need to explore further the assignment of values to lower order bits of $b$ to separate those assignments that contribute to $n_{0}$ from those that contribute to $n_{1}$. To do this, we will symbolically reduce the major and minor $(m-1)$-equations to their modes for this choice of $b_{m-1}$, and proceed to the equations involving $b_{m-2}$.

The reason behind the reduction of component equations to behavior modes becomes clear if we consider the situation when we are considering how the assignment of values to $b_{i}$, with $0 \leqslant i<m-1$, affects the counts $n_{0}$ and $n_{1}$. The fact that we are reasoning about $b_{i}$ means: 
- that we have already considered the bits $b_{m-1}$ through $b_{i+1}$;

- that we have identified the unique assignment of values of these bits that leads to $P$-modes for the major $(m-1)$-equation through the major $(i+1)$-equation;

- that we have reduced all of these major and minor equations to their appropriate behavior modes for these assignments of values to bits $b_{m-1}$ through $b_{i+1}$.

If component equation $t$ is the major $i$-equation, then this means that component equations $2 m-1$ through $t+1$ have been reduced. (Some of the component equations $t-1$ through 0 may also have been reduced; this does not concern us yet, because carries move from lower order to higher order bits.) In any case, one of the two choices of $b_{i}$ will lead to a $G_{\epsilon}$ mode for component equation $t$. However, we cannot at this point simply increment $n_{\epsilon}$ by $2^{i-1}$, since the generated carry $k_{t+1}$ may be altered as it travels through the reduced component equations $t+1$ through $m-1$. What we need to do is to determine the value $k_{2 m}=\delta$ that emerges at the other end of this process, and increment $n_{\delta}$ by $2^{i-1}$. The representation of component equations as modes facilitates the determination of $k_{2 m}$.

One final observation about the algebraic structure of modes allows us to calculate the terminal carry $k_{2 m}$ in a constant number of operations. It is easily seen that the mode set $\left\{P, G_{0}, G_{1}\right\}$ is a monoid under composition, with $P$ as the identity element. Composition is defined by the following table.

\begin{tabular}{c|ccc} 
& $P$ & $G_{0}$ & $G_{1}$ \\
\hline$P$ & $P$ & $G_{0}$ & $G_{1}$ \\
$G_{0}$ & $G_{0}$ & $G_{0}$ & $G_{0}$ \\
$G_{1}$ & $G_{1}$ & $G_{1}$ & $G_{1}$
\end{tabular}

In trying to interpret this "composition table," remember that carries move from right to left. Thus, $G_{1} P$ means that an input carry first passes through a $P$-mode and then through a $G_{1}$-mode. This is equivalent to a $G_{1}$-mode. Similarly, $G_{1} G_{0}$ produces a $G_{1}$-mode, because an input carry first passes through a $G_{0}$-mode, producing a carry value of 0 , which then passes through a $G_{1}$-mode, producing an output carry of 1 . Given this monoid structure, instead of maintaining individual modes for reduced component equations $i+1$ through $2 m-1$ and laboriously propagating $k_{t+1}$ through them to obtain $k_{2 m}$, we can keep a compact description of the combined effect of these modes and obtain $k_{2 m}$ from $k_{t+1}$ in a single step. Furthermore, we can incrementally update this description as we move to lowernumbered component equations.

We are now ready to present the complete algorithm to determine $\left(n_{0}, n_{1}\right)$.

$$
\begin{array}{ll}
1 & n_{0} \leftarrow 0 \\
2 & n_{1} \leftarrow 0 \\
3 & \text { mode } \leftarrow P \\
4 & i \leftarrow m-1
\end{array}
$$


5 for $t=2 m-1$ downto 0 do

6 if component equation $t$ has been reduced to mode $M$ then else $/ *$ This is the major $i$-equation */

$v \leftarrow$ value of $b_{i}$ that makes this equation behave in mode $G_{\epsilon}$, from Lemma 2.2

Locate the minor $i$-equation and reduce it to the mode resulting from setting $b_{i}=\bar{v}$

$$
13 \quad i \leftarrow i-1
$$

\section{4 endif}

\section{5 enddo}

$16 \delta \leftarrow \operatorname{APPLY}\left(\operatorname{mode}, k_{0}\right)$

$17 n_{\delta} \leftarrow n_{\delta}+1$

THEOREM 2.1. The above program correctly computes $n_{0}$ and $n_{1}$ and runs in $O(m)$ steps.

Proof. Immediate from Lemmas 2.1 and 2.2.

EXAmPLE 3. Let $\sigma=001110$, let $d_{2 m-1} \cdots d_{0}=011000$ and let $k_{0}=1$. In this case, the equations are

$$
\begin{array}{ll}
E_{0}: & a_{0}=b_{0}+0 \\
E_{1}: & b_{0}=c_{0}+0 \\
E_{2}: & b_{1}=c_{1}+0 \\
E_{3}: & b_{2}=c_{2}+1 \\
E_{4}: & a_{1}=b_{1}+1 \\
E_{5}: & a_{2}=b_{2}+0 .
\end{array}
$$

The system of equations and $\left(n_{0}, n_{1}\right)$ evolve in the following way as we go through the steps of the algorithm.

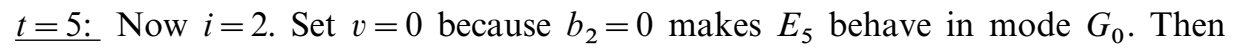
$\delta=0$ because mode $=P$ and $\epsilon=0$ (i.e., a carry of 0 propagates through the reduced component equations). Update $n_{0}=0+4 . E_{3}$ is the minor 2-equation and gets reduced to $P$-mode. This leaves

$$
\begin{array}{ll}
E_{0}: & a_{0}=b_{0}+0 \\
E_{1}: & b_{0}=c_{0}+0 \\
E_{2}: & b_{1}=c_{1}+0
\end{array}
$$




$$
\begin{array}{lll}
E_{3}: & b_{2}=c_{2}+1 & P \text {-mode } \\
E_{4}: & a_{1}=b_{1}+1 & \\
E_{5}: & a_{2}=b_{2}+0 & P \text {-mode }
\end{array}
$$

$t=4$ : Now $i=1$. Set $v=1$ and $\delta=1$. Update $n_{1}=0+2 . E_{2}$ is the minor 1-equation and gets reduced to $P$-mode. This leaves

$$
\begin{aligned}
& E_{0}: a_{0}=b_{0}+0 \\
& E_{1}: b_{0}=c_{0}+0 \\
& E_{2}: \quad b_{1}=c_{1}+0 \quad P \text {-mode } \\
& E_{3}: \quad b_{2}=c_{2}+1 \quad P \text {-mode } \\
& E_{4}: \quad a_{1}=b_{1}+1 \quad P \text {-mode } \\
& E_{5}: a_{2}=b_{2}+0 \quad P \text {-mode }
\end{aligned}
$$

$\underline{t=3}$ : mode $=P$ because the previous value of mode, $P$, composed with the mode of $E_{3}, P$, is $P$.

$\underline{t=2:}$ mode $=P$.

$\underline{t=1}$ : Now $i=0$. Set $v=1$ and $\delta=0$. Update $n_{0}=4+1 . E_{0}$ is the minor 0 -equation and gets reduced to $G_{0}$-mode. This leaves

$$
\begin{array}{lll}
E_{0}: & a_{0}=b_{0}+0 & G_{0} \text {-mode } \\
E_{1}: & b_{0}=c_{0}+0 & P \text {-mode } \\
E_{2}: & b_{1}=c_{1}+0 & P \text {-mode } \\
E_{3}: & b_{2}=c_{2}+1 & P \text {-mode } \\
E_{4}: & a_{1}=b_{1}+1 & \text { P-mode } \\
E_{5}: & a_{2}=b_{2}+0 & P \text {-mode }
\end{array}
$$

$\underline{t=0}:$ mode $=G_{0}$. Set $\delta=0$ and update $n_{0}=5+1$.

The final values for $\left(n_{0}, n_{1}\right)$ are $(6,2)$, which agrees with the answer obtained by explicit generation of all solutions. So, if $d_{\rho-1}=\cdots=d_{2 m}=0$ then $\mathbf{A B}(\mathbf{d})=6$, whereas if $d_{\rho-1}=\cdots=d_{2 m}=1$ then $\mathbf{A B}(\mathbf{d})=2$.

\subsection{Algorithm $\mathrm{AC}(d)$}

We now investigate the following problem: Given an $(m, m)$-interleaving $\sigma$, a nonnegative integer $d$ with a $\rho$-bit binary expansion, determine $\mathbf{A C}(\mathbf{d})$, the number of triples $(a, b, c) \in B_{m}^{3}$ such that

$$
\Theta(a, b)=\Theta(a, c)+d \bmod 2^{\rho}
$$


under the condition that $2 m \leqslant \rho$. This problem is superficially similar to Eq. (2), with one small but critical difference: the variable that occurs on both sides of Eq. (10) occurs in the 0 -positions of $\sigma$ on both sides of the equation, whereas the variable that occurs on both sides of Eq. (2) occurs in the 0-position of $\sigma$ on one side of the equation and the 1-position of $\sigma$ on the other side of the equation. This difference makes the combinatorics of Eq. (10) radically different from the combinatorics of Eq. (2), leading in the end to a conceptually simpler algorithm to compute $\mathbf{A C}(\mathbf{d})$.

If we write out Eq. (10) in terms of component bit-equations as we did for Eq. (2), we see that component equation $t$ (for $0 \leqslant t<2 m$ ) has one of two forms: $a_{i}=a_{i}+d_{t}$ if $\sigma_{t}=0$, and $b_{i}=c_{i}+d_{t}$ if $\sigma_{t}=1$. The decoupling of the bits of $a$ from the bits of $b$ and $c$ indicates that the $a$-component of any solution of Eq. (10) can be chosen independent of the $b$ - and $c$-components. The decoupling also suggests that we need to look at the distribution of $0 \mathrm{~s}$ and $1 \mathrm{~s}$ in $\sigma$. Based on these observations, we start with a few definitions.

Definition 2.2 (Runs of $\sigma$ ). Let $\sigma$ be an $(m, m)$-interleaving, and let $P$ be the sequence $[0, \ldots, \rho-1]$. For $\epsilon \in\{0,1\}$, an $\epsilon$-run of $\sigma$ is a maximal-length contiguous subsequence $[u, \ldots, v]$ of $P$ such that $\sigma_{u}=\cdots=\sigma_{v}=\epsilon$, where $\sigma_{2 m}$ through $\sigma_{\rho-1}$ are declared to be 0 . Order $\epsilon$-runs in increasing order of $u$, and denote the $i$ th $\epsilon$-run of $\sigma$ by $R_{i}^{(\epsilon)}$.

For technical reasons that will soon become evident, we will always want the "lowest" run to be a 0 -run. This is a problem only when $\sigma_{0}=1$. In this case, we will create a special empty 0 -run $R_{1}^{(0)}$ and label the nonempty 0-runs from $R_{2}^{(0)}$ onward. Thus, $R_{i}^{(1)}$ is sandwiched between $R_{i}^{(0)}$ and $R_{i+1}^{(0)}$. Note also that the 0 -runs constrain possible choices of $a$, while the 1-runs constrain possible choices of $b$ and $c$.

We obtain strong conditions on the (non)existence of solutions of Eq. (10) by considering the restrictions of $d$ to the 0-runs of $\sigma$. The intuition behind the following lemma and its proof are small variations of Lemma 2.1 .

LEMMA 2.3. Equation (10) has no solutions if $d$ is inconsistent on any 0-run of $\sigma$. For $\epsilon \in\{0,1\}$, if $d$ is $\epsilon$-consistent on $R_{i}^{(0)}=[u, \ldots, v]$, then Eq. (10) has solutions iff $k_{u}=k_{v+1}=\epsilon$. If $R_{1}^{(0)}$ is empty, then every $d$ is declared to be 0 -consistent on it.

Lemma 2.3 has two important consequences. First, it provides an early termination test for the algorithm. Second, if $d$ is indeed consistent on all 0-runs of $\sigma$, then it simplifies the counting of the number of choices of $a$ in the following way. Note that each of the component equations is of the form $a_{i}=a_{i}+d_{t}$. Since the same element of $a$ appears on both sides of the equation, there is in fact no constraint on $a$ ! Thus, for every possible choice of $b$ and $c$ that we discover by examining the 1 -runs (which we will do shortly), any of the $2^{m}$ choices of $a$ will work.

Consider $R_{i}^{(1)}=[u, \ldots, v]$, the $i$ th 1 -run of $\sigma$. Recall that this run is sandwiched between runs $R_{i}^{(0)}$ and $R_{i+1}^{(0)}$. Let $d$ be $z_{u}$-consistent on $R_{u}^{(0)}$. Let $t=\left|R_{1}^{(1)}\right|+\cdots+$ $\left|R_{i-1}^{(1)}\right|$. Then the component equations in $R_{i}^{(1)}$ are

$$
\begin{aligned}
b_{t} & =c_{t} \oplus d_{u} \oplus k_{u} \\
k_{u+1} & =\operatorname{MAJ}\left(c_{t}, d_{u}, k_{u}\right)
\end{aligned}
$$




$$
\begin{aligned}
b_{t+1} & =c_{t+1} \oplus d_{u+1} \oplus k_{u+1} \\
k_{u+2} & =\operatorname{MAJ}\left(c_{t+1}, d_{u+1}, k_{u+1}\right) \\
& \ldots \\
b_{t+v-u} & =c_{t+v-u} \oplus d_{v} \oplus k_{v} \\
k_{v+1} & =\operatorname{MAJ}\left(c_{t+v-u}, d_{v}, k_{v}\right) .
\end{aligned}
$$

By Lemma 2.3, we know that $k_{u}=z_{i}$ and $k_{v+1}=z_{i+1}$. Thus we are constrained by being given the values of both the initial and terminal carries of the 1-run, and must determine how many choices of bit values for $b$ and $c$ honor these constraints. It turns out that the easiest way to count the possibilities is to reason about the bit patterns as nonnegative integers. To this end, define $\delta_{i}=z_{u}+\sum_{j=u}^{v} d_{j} 2^{j-u}$. That is, $\delta_{i}$ is the integer corresponding to the bit pattern $d_{v} \cdots d_{u}$, with the initial carry value absorbed into it. Also, let $\Delta_{i}=2^{v-u+1}-\delta_{i}$. We then get the following result by case analysis on the value of $k_{v+1}$.

THEOREM 2.2. Let $\sigma$ be an $(m, m)$-interleaving and let $d \in B_{\rho}$ be consistent on all 0 -runs of $\sigma$. Then the number of solutions to Eq. (10) is $2^{m} \cdot \prod_{i=1}^{\ell} F_{i}$, where $\ell$ is the number of 1-runs of $\sigma$ and

$$
F_{i}= \begin{cases}\Delta_{i}, & \text { if } d \text { is } 0 \text {-consistent on } R_{i+1}^{(0)} \\ \delta_{i}, & \text { if } d \text { is } 1 \text {-consistent on } R_{i+1}^{(0)}\end{cases}
$$

Proof. By equating the coefficients of the distinct powers of 2 on the two sides of (10) we arrive at a set of restrictions on the sequences $a, b, c$. Lemma 2.3 describes restrictions that result from equating coefficients of powers $2^{\tau}$ where $\tau$ is in a 0-run of $\sigma$. The elements of $a$ appear in these equations, with the same element of $a$ appearing on both sides. This gives no restrictions on $a$ and so there are $2^{m}=n$ choices for $a$. This accounts for the factor of $2^{m}$ that appears in the formula. The remaining factors will count the number of choices we have for $b$ and $c$.

Consider restrictions on $b$ and $c$ that result from equating coefficients of powers $2^{\tau}$ for $\tau$ in a particular 1-run $R_{i}^{(1)}$. Define $\beta_{i}$ and $\gamma_{i}$ by $\beta_{i}=\sum_{j=t}^{t+v-u} b_{j} 2^{j-t}$ and $\gamma_{i}=\sum_{j=t}^{t+v-u} c_{j} 2^{j-t}$.

Case 1. Suppose $z_{i+1}=0$. Then the component equations on $R_{i}^{(1)}$ are equivalent to

$$
\beta_{i}-\gamma_{i}=\delta_{i}
$$

where we have equality of integers in Eq. (11). So, the number of choices we have for $b_{j}, c_{\ell}$ satisfying the component equation on $R_{i}^{(1)}$ is equal to the number of integers $\beta_{i}, \gamma_{i}$ with $0 \leqslant \beta_{i}<2^{v-u+1}, 0 \leqslant \gamma_{i}<2^{v-u+1}$ that satisfy Eq. (11). For each $\beta_{i}$ with $\delta_{i} \leqslant \beta_{i}<2^{v-u+1}$ there is exactly one choice of $\gamma_{i}$ such that $\beta_{i}, \gamma_{i}$ satisfy 
Eq. (11). For $0 \leqslant \beta_{i}<\delta_{i}$ there are no choices of $\gamma_{i}$ such that $\beta_{i}, \gamma_{i}$ satisfy Eq. (11). So the number of solutions to Eq. (11) is

$$
2^{v-u+1}-\delta_{i}=\Delta_{i},
$$

which is the $i$ th factor in the product in the statement of the theorem.

Case 2. Suppose $z_{i+1}=1$. Then the component equations on $R_{i}^{(1)}$ are equivalent to

$$
\beta_{i}+2^{v-u+1}=\delta_{i}+\gamma_{i}
$$

which can be rewritten as

$$
\gamma_{i}-\beta_{i}=2^{v-u+1}-\delta_{i}
$$

By the same reasoning as above, the number of solutions to Eq. (12) is $\delta_{i}$, which is the $i$ th factor in the product in the statement of the theorem.

EXAMPLE 4. Let $m=5, \rho=12, \sigma=0110110001$, and $d=111100101111$. We will use Algorithm AC to compute $\mathbf{A C}(\mathbf{d})$.

In Step 1 we compute the runs and the consistency values $z_{i}$

$$
\begin{aligned}
& R_{1}^{(0)}=[], z_{1}=0 \\
& R_{1}^{(1)}=[0] \\
& R_{2}^{(0)}=[1,2,3], z_{2}=1 \\
& R_{2}^{(1)}=[4,5] \\
& R_{3}^{(0)}=[6], z_{3}=0 \\
& R_{3}^{(1)}=[7,8] \\
& R_{4}^{(0)}=[9,10,11], z_{4}=1 .
\end{aligned}
$$

In Step 2 we compute the factors $F_{i}$ and use them to determine $\mathbf{A C}(\mathbf{d})$

$$
\begin{array}{cccc}
i & \delta_{i} & \Delta_{i} & F_{i} \\
1 & 1 & 1 & 1 \\
2 & 3 & 1 & 1 \\
3 & 2 & 2 & 2
\end{array}
$$

So $\mathbf{A C}(\mathbf{d})=32 \cdot 1 \cdot 1 \cdot 2=64$.

\subsection{Counting Joint Solutions}

The last problem we will consider in this section is to count those triples $a, b, c \in B_{m}$ which satisfy the two equations

$$
\Theta(a, b)=\Theta(b, c)+d
$$


and

$$
\Theta(a, b)=\Theta(a, c)+e
$$

simultaneously. It is instructive to consider an example.

ExAmPle 5. Let $m=5, \rho=11, \sigma=0110001011, d=00010101111$, and $e=$ 00110001101. Recalling the characteristic sequence notation from Section $1.3, \chi_{\sigma}=$ $f_{4} s_{4} s_{3} f_{3} f_{2} f_{1} s_{2} f_{0} s_{1} s_{0}$. Then the simultaneous equations that must be satisfied are

$$
\begin{aligned}
& \Theta(a, b)=\Theta(b, c)+d \quad \Theta(a, b)=\Theta(a, c)+e \\
& b_{0}=c_{0}+1 \quad b_{0}=c_{0}+1 \quad s_{0} \\
& b_{1}=c_{1}+1+k_{0} \quad b_{1}=c_{1}+0+\ell_{0} \quad s_{1} \\
& a_{0}=b_{0}+1+k_{1} \quad a_{0}=a_{0}+1+\ell_{1} \quad f_{0} \\
& b_{2}=c_{2}+1+k_{2} \quad b_{2}=c_{2}+1+\ell_{2} \quad s_{2} \\
& a_{1}=b_{1}+0+k_{3} \quad a_{1}=a_{1}+0+\ell_{3} \quad f_{1} \\
& a_{2}=b_{2}+1+k_{4} \quad a_{2}=a_{2}+0+\ell_{4} \quad f_{2} \\
& a_{3}=b_{3}+0+k_{5} \quad a_{3}=a_{3}+0+\ell_{5} \quad f_{3} \\
& b_{3}=c_{3}+1+k_{6} \quad b_{3}=c_{3}+1+\ell_{6} \quad s_{3} \\
& b_{4}=c_{4}+0+k_{7} \quad b_{4}=c_{4}+1+\ell_{7} \quad s_{4} \\
& a_{4}=b_{4}+0+k_{8} \quad a_{4}=a_{4}+0+\ell_{8} \quad f_{4} \\
& 0=0+k_{9} \quad 0=0+\ell_{9}
\end{aligned}
$$

In the above set of equations, $k_{t}$ is the carry from the $t$ th to $(t+1)$ st equation in $\Theta(a, b)=\Theta(b, c)+d$ whereas $\ell_{t}$ is the carry from the $t$ th to the $(t+1)$ st equation in $\Theta(a, b)=\Theta(a, c)+e$. We will refer to these two sets of equations as the $d$-system and the e-system. Also, we will let $B$ denote the number of $f_{i}$-equations in the system above. Note that $B=m$ if $2 m \leqslant \rho$.

As we will see, it is seldom the case that there are any simultaneous solutions to Eqs. (13) and (14). The next result states that even if there are simultaneous solutions, there are not very many.

THEOREM 2.3. The number of simultaneous solutions to Eqs. (13) and (14) is less than or equal to $2^{-B}$ times the number of solutions to Eq. (14).

Proof. Suppose there is a simultaneous solution to Eqs. (13) and (14). Then the $s_{i}$-equations determine the values of $b_{0}, b_{1}, \ldots, b_{B-1}$. To this simultaneous solution of Eqs. (13) and (14) we can correspond $2^{B}$ solutions to Eq. (14), which have the same $b_{i}$ and $c_{i}$ but where the choices of $a_{0}, a_{1}, \ldots, a_{B-1}$ range over all possibilities. 
One might ask whether there are instances in which the number of simultaneous solutions to Eqs. (13) and (14) is exactly $2^{-B}$ times the number of solutions to Eq. (14). The next result tells us that this the case when $d=e$.

Definition 2.3. Let $S$ denote the set of solutions to Eq. (14). We say two solutions $\left(a^{(1)}, b^{(1)}, c^{(1)}\right)$ and $\left(a^{(2)}, b^{(2)}, c^{(2)}\right) \in S$ are equivalent if $b^{(1)}=b^{(2)}$ and $c^{(1)}=c^{(2)}$.

It is straightforward to see that every equivalence class has size $n$ and that equivalence classes are indexed by pairs $b, c \in B_{m}$.

THEOREM 2.4. If $d=e$, then there is exactly one solution to Eq. (13) in every equivalence class of solutions to Eq. (14).

Proof. Consider the equivalence class indexed by the pair $b, c$. It is clear that there is at most one solution $(a, b, c)$ to Eq. (13) in that equivalence class because $a_{i}$ is determined by equation $f_{i}$. It remains to show that there is at least one solution.

Consider the process of solving for the $a_{i}$ and the carries $k_{t}$ in equations of type (13) starting with $b, c$ which gives (along with any $a$ ) a solution to Eq. (14). The thing we need to check is that the carries $k_{t}$ we get in the equations of type (13) are identical to the carries $\ell_{t}$ we get in equations of type (14). We see this by induction on $t$.

Assume that $k_{t-1}=\ell_{t-1}$. There are two cases to consider. First assume equation $t$ is labeled $s_{i}$ so that in system (13) the $t$ th equation is $b_{i}=c_{i}+d_{t}+k_{t-1}$ and in system (14) the $t$ th equation is $b_{i}=c_{i}+d_{t}+\ell_{t-1}$. In this situation it is clear that $k_{t}$ will be equal to $\ell_{t}$. Next, assume that equation $t$ is labeled $f_{i}$. In this case the $t$ th equation in system (13) is $a_{i}=b_{i}+d_{t}+k_{t-1}$, whereas the $t$ th equation in system (14) is $a_{i}=a_{i}+d_{t}+\ell_{t-1}$. By Lemma 2.3 we have $\ell_{t}=\ell_{t-1}=d_{t}$. By our induction hypothesis, $k_{t-1}=\ell_{t-1}=d_{t}$. Because $k_{t-1}=d_{t}$ we have $k_{t}=d_{t}$ so $k_{t}=\ell_{t}$, which completes the induction step and finishes the proof.

\section{Corollary 2.5. Let notation be as in Theorem 2.3. Then:}

1. The number of triples $(a, b, c)$ which are simultaneous solutions to $\Theta(a, b)=\Theta(b, c)+d$ and $\Theta(a, b)=\Theta(a, c)+d i s \prod F_{i}$.

2. The number of simultaneous solutions can be computed in $O(\rho)$ steps.

The above results show that there are not very many simultaneous solutions of Eqs. (13) and (14). The next results indicate that in most instances there are no simultaneous solutions.

Suppose there exist simultaneous solutions to Eqs. (13) and (14). From our previous analysis, we know a number of things.

(a) $e$ must be consistent on 0-runs of $\sigma$.

(b) In Eq. (14) the carry into any 0-run and carry out of that 0-run must both match the value of $e$ on that run.

As a first test to whether there exist simultaneous solutions to Eqs. (13) and (14), conditions (a) and (b) can be checked in $O(\rho)$ steps. We are now going to focus on 1-runs. 
Suppose that equations $u, u+1, \ldots, u+j-1$ constitute a 1-run and that these equations are labeled $s_{i}, s_{i+1}, \ldots, s_{i+j-1}$. Let $\beta, \gamma, \delta, \varepsilon$ be the numbers with binary expansions given

$$
\begin{aligned}
& \beta=b_{i+j-1} \cdots b_{i+1} b_{i} \\
& \gamma=c_{i+j-1} \cdots c_{i+1} c_{i} \\
& \delta=d_{u+j-1} \cdots d_{u+1} d_{u} \\
& \varepsilon=e_{u+j-1} \cdots e_{u+1} e_{u} .
\end{aligned}
$$

By comparing equations $s_{i}, \ldots, s_{i+j-1}$ in Eqs. (13) and (14) we see that

$$
\beta=\gamma+\delta+k_{u-1}=\gamma+\varepsilon+\ell_{u-1}
$$

Also, $\ell_{u-1}$ is specified to be the consistent value of $e$ on preceding 0 -run and $\gamma$ must be chosen so that $\gamma+\varepsilon+\ell_{u-1}$ is less than $2^{j}$ iff the value on $e$ on the subsequent 0 -run is 0 . From Eq. (15), the following result follows immediately.

THEOREM 2.6. Let $\delta$ and $\varepsilon$ be the numbers whose binary expansions are given by the binary digits of $d$ and $e$ on a 1-run of $\sigma$ as above. If there are simultaneous solutions to Eqs. (13) and (14) then $\delta$ and $\varepsilon$ must differ by no more than 1.

More precisely, we must have one of the following four cases:

1. $\delta=\varepsilon+\ell_{u-1}$. Note. In this case we also must have $k_{u-1}=0$.

2. $\delta=\varepsilon+\ell_{u-1}-1$. Note. In this case we also must have $k_{u-1}=1$.

3. $\delta=0, \varepsilon=2^{j}-1, \ell_{u-1}=1$. Note. In this case, we also must have that $k_{u-1}=0$ and that $e$ is consistently 1 on the next 0 -run.

4. $\delta=2^{j}-1, \varepsilon=\ell_{u-1}=0$. Note. In this case, we also must have that $k_{u-1}=1$ and that $e$ is consistently 0 on the next 0 -run.

Theorem 2.6 gives another $O(\rho)$ test which can determine that there are no simultaneous solutions to Eqs. (13) and (14). Note that if we assume $d$ and $e$ are chosen randomly, then Theorem 2.6 together with condition (a) show that the probability that there exist simultaneous solutions to Eqs. (13) and (14) is no more than $2^{-(B+U-R)}$, where $B$ is the number of $f_{i}$-equations, $U$ is the number of $s_{i}$-equations and $R$ is the number of runs of $\sigma$. Note that $B+U=\min \{2 m, \rho\}$. Alternatively, if we have some freedom to choose $d, e$ and $\sigma$, then the conditions given in (a) and Theorem 2.6 can be used to insure that there are no simultaneous solutions to Eqs. (13) and (14). We will return to this important point in our later paper on minimizing the number of cache misses.

It seems unlikely to us that there exists an algorithm which is polynomial in $m$ or linear in $\rho$ which determines the exact number of simultaneous solutions to Eqs. (13) and (14). Just to conclude, we examine the case given in Example 5 just to point out some of the complexities of this problem. 
Turning to the set of equations given in Example 5, we first examine whether the conditions set out in (a) and (b) hold. It can be seen that $e$ is consistent on 0-runs with value 1 on $f_{0}$, value 0 on $f_{1}, f_{2}, f_{3}$ and value 1 on $f_{4}$. Condition (b) thus implies that $\ell_{1}=\ell_{2}=1, \ell_{3}=\ell_{4}=\ell_{5}=\ell_{6}=0$ and $\ell_{8}=\ell_{9}=0$.

To now consider the constraints given by Theorem 2.6, we must look at 1-runs. For the 1-run $s_{0}, s_{1}$, we have $\delta=\varepsilon=3$ and $\ell_{-1}=0$. So we are in Case 1. This implies that $k_{-1}=0$ and that $\ell_{1}=1$. This gives a constraint on $\gamma=c_{0} c_{1}$ i.e., $\gamma+\varepsilon \geqslant 4$. This constraint on $\gamma$, which comes from consideration of the $e$ equations, which implies that $k_{1}=1$.

Moving now to the 1 -run $s_{2}$, we have that $\delta=1, \varepsilon=0$ and $l_{2}=1$. So we are again in Case 1 which implies that $k_{2}=0$. But now we have an inconsistency: it is impossible to have $k_{1}=1$ and $k_{2}=0$. So there are no simultaneous solutions to Eqs. (13) and (14) in the case given in Example 5.

This particular example gives a flavor for the complex interplay that can take place between the constraints imposed by the $d$ equations and those imposed by the $e$ equations. At this time, we do not know a fast algorithm to determine the number of simultaneous solutions exactly.

\section{INCORPORATING CACHE BLOCK SIZE}

In the last section, we devised fast algorithms to compute the number of solutions to systems of equations of the form

$$
\Theta(a, b)=\Theta(b, c)+d \bmod 2^{\rho}
$$

and

$$
\Theta(a, b)=\Theta(a, c)+d \bmod 2^{\rho} .
$$

In practice, we will need to extend these algorithms to enumerate solutions to a slightly different pair of equations. Usually $2^{\lambda}$ memory locations fit into a cache block, represented by the denominator in the following equations.

Thus, the equation has to be taken $\bmod 2^{\rho-\lambda}$. In practice, $\lambda$ often equals 2 or 4 . We show the case $\lambda=2$ to provide the case distinction in full detail; the extension for $\lambda \in \mathbb{N}_{0}^{+}$is straightforward, but requires consideration of more cases for $\lambda>2 .^{3}$

$$
\left\lfloor\frac{\Theta(a, b)+\alpha}{4}\right\rfloor=\left\lfloor\frac{\Theta(b, c)+\beta}{4}\right\rfloor \bmod 2^{\rho-2}
$$

and

$$
\left\lfloor\frac{\Theta(a, b)+\alpha}{4}\right\rfloor=\left\lfloor\frac{\Theta(a, c)+\beta}{4}\right\rfloor \bmod 2^{\rho-2},
$$

where $\alpha, \beta \in B_{\rho}$.

\footnotetext{
${ }^{3}$ In general, we need to determine the distribution of output carry values at bit $\lambda-1$. This can be accomplished in a preprocessing step that takes $O\left(\lambda^{2}\right)$ operations.
} 
In this section we sketch methods, based on the ideas and algorithms developed in Section 2, to compute the number of solutions to Eqs. (16) and (17). We will take the two equations in turn, starting with Eq. (17) because much of what we find there can later be reused for the treatment of Eq. (16).

\subsection{Computing the Number of Solutions to Eq. (17)}

To begin, we will write out the digits in the binary expansions of $\Theta(a, b)+\alpha$ and $\Theta(a, c)+\beta$. Equating these expressions gives a system of equations $\underline{E}=E_{0}, E_{1}, \ldots$, $E_{\rho-1}$, where Eq. (17) imposes the requirement that equations $E_{2}, E_{3}, \ldots, E_{\rho-1}$ must be satisfied $\bmod 2$. In order to satisfy Eq. (17), $E_{0}$ or $E_{1}$ need not hold mod 2. Consider $E_{0}$ and $E_{1}$. They look like one of the following

$$
\begin{gathered}
a_{0}+\alpha_{0}=a_{0}+\beta_{0} \\
a_{1}+\alpha_{1}+k_{1}=a_{1}+\beta_{1}+\ell_{1}
\end{gathered}
$$

or

$$
\begin{gathered}
a_{0}+\alpha_{0}=a_{0}+\beta_{0} \\
b_{0}+\alpha_{1}+k_{1}=c_{0}+\beta_{1}+\ell_{1}
\end{gathered}
$$

or

$$
\begin{gathered}
b_{0}+\alpha_{0}=c_{0}+\beta_{0} \\
a_{0}+\alpha_{1}+k_{1}=a_{0}+\beta_{1}+\ell_{1}
\end{gathered}
$$

or

$$
\begin{gathered}
b_{0}+\alpha_{0}=c_{0}+\beta_{0} \\
b_{1}+\alpha_{1}+k_{1}=c_{1}+\beta_{1}+\ell_{1},
\end{gathered}
$$

where $k_{1}$ is the carry from the left side of $E_{0}$, and $\ell_{1}$ is the carry from the right side of $E_{0}$. Case (18) occurs when $\sigma_{1} \sigma_{0}=00$, (19) when $\sigma_{1} \sigma_{0}=10,(20)$ when $\sigma_{1} \sigma_{0}=01$, and (21) when $\sigma_{1} \sigma_{0}=11$. The key observation is that the variables which appear in these equations do not appear in any of the later equations $E_{2}, E_{3}, \ldots, E_{\rho-1}$, because only $a_{i}$ can occur more than once for each $i$ and both instances of $a_{i}$ are in equation $E_{z}$, where $z$ is the bit position to which $\sigma$ maps $a_{i}$.

Our algorithm for enumerating the solutions to Eq. (17) begins with a loop over all possible choices of values for the variables that occur in $E_{0}$ and $E_{1}$. So, this outer loop runs through $4,8,8$, or 16 possibilities depending on whether we are in case (18), (19), (20), or (21) respectively.

Once values for these variables have been chosen, we compute the carries $k_{2}$ and $\ell_{2}$ that are added to the left and right sides of $E_{2}$. Let

$$
\alpha^{\prime}=k_{2}+\sum_{i=2}^{\rho-1} \alpha_{i} 2^{i-2}
$$


and

$$
\beta^{\prime}=\ell_{2}+\sum_{i=2}^{\rho-1} \beta_{i} 2^{i-2}
$$

Then the number of solutions to Eq. (17) with the chosen values for the variables in $E_{0}$ and $E_{1}$ is equal to the number of solutions to

$$
\Theta^{\prime}\left(a^{\prime}, b^{\prime}\right)+\alpha^{\prime}=\Theta^{\prime}\left(a^{\prime}, c^{\prime}\right)+\beta^{\prime} \bmod 2^{\rho-2},
$$

where $a^{\prime}, b^{\prime}, c^{\prime}$ each come from $B_{m-2}, B_{m-1}$ or $B_{m}$ depending on whether $\sigma_{1} \sigma_{0}=00,10,01$ or 11 . Here $\Theta^{\prime}$ is the mixing function based on the interleaving $\sigma^{\prime}$ obtained from $\sigma$ by deleting $\sigma_{0}$ and $\sigma_{1}$. Let

$$
d= \begin{cases}\beta^{\prime}-\alpha^{\prime} & \text { if } \beta^{\prime} \geqslant \alpha^{\prime} \\ 2^{\rho-2}+\beta^{\prime}-\alpha^{\prime} & \text { if } \beta^{\prime}<\alpha^{\prime}\end{cases}
$$

Then the number of solutions to equation (22) is equal to the number of solutions to

$$
\Theta^{\prime}\left(a^{\prime}, b^{\prime}\right)=\Theta^{\prime}\left(a^{\prime}, c^{\prime}\right)+d,
$$

which can be computed using the AC algorithm in $O(m+\rho)$ steps.

With this generalization we call the algorithm the extended AC Algorithm.

\subsection{Computing the Number of Solutions to Eq. (16)}

As in Section 3.1 we will begin by writing out expressions for the digits in the binary expansions of $\Theta(a, b)+\alpha$ and $\Theta(b, c)+\beta$. This gives a system of equations $E_{0}, E_{1}, \ldots, E_{\rho-1}$, where the requirement of Eq. (16) is that $E_{2}, \ldots, E_{\rho-1}$ must be satisfied $\bmod 2\left(E_{0}\right.$ and $E_{1}$ need not hold $\left.\bmod 2\right)$.

Again, we will look at $E_{0}, E_{1}$ and find that they have one of four possible forms

$$
\begin{gathered}
a_{0}+\alpha_{0}=b_{0}+\beta_{0} \\
a_{1}+\alpha_{1}+k_{1}=b_{1}+\beta_{1}+\ell_{1}
\end{gathered}
$$

or

$$
\begin{gathered}
a_{0}+\alpha_{0}=b_{0}+\beta_{0} \\
b_{0}+\alpha_{1}+k_{1}=c_{0}+\beta_{1}+\ell_{1}
\end{gathered}
$$

or

$$
\begin{gathered}
b_{0}+\alpha_{0}=c_{0}+\beta_{0} \\
a_{0}+\alpha_{1}+k_{1}=b_{0}+\beta_{1}+\ell_{1}
\end{gathered}
$$


or

$$
\begin{gathered}
b_{0}+\alpha_{0}=c_{0}+\beta_{0} \\
b_{1}+\alpha_{1}+k_{1}=c_{1}+\beta_{1}+\ell_{1},
\end{gathered}
$$

where $k_{1}$ is the carry from the left side of $E_{0}$, and $\ell_{1}$ is the carry from the right side of $E_{0}$. Case (23) occurs when $\sigma_{1} \sigma_{0}=00,(24)$ when $\sigma_{1} \sigma_{0}=10,(25)$ when $\sigma_{1} \sigma_{0}=01$, and (26) when $\sigma_{1} \sigma_{0}=11$. Note that in (24) and (25) the variables which occur in equations $E_{0}$ and $E_{1}$ do not occur in $E_{2}, E_{3}, \ldots, E_{\rho-1}$, because only $b_{i}$ can occur more than once for each $i$ and both instances of $b_{0}$ occur in $E_{0}$ and $E_{1}$. Therefore, we can use the same method we used in Section 3.1 to devise fast algorithms to compute the number of solutions.

Cases (23) and (26) are slightly different because $b_{0}$ and $b_{1}$ may occur later in $E_{2}, E_{3}, \ldots, E_{\rho-1}$. In Case (23), our algorithm has an outside loop over the four possible choices of values for $a_{0}$ and $a_{1}$. For each choice of these values, we compute the carry $k_{2}$ which is added to the left-hand side of $E_{2}$. We let

$$
\alpha^{\prime}=4 k_{2}+\sum_{i=2}^{\rho-1} \alpha_{i} 2^{i}
$$

and we apply the AB Algorithm to count the number of solutions to

$$
\Theta(a, b)=\Theta(b, c)+d
$$

where

$$
d= \begin{cases}\beta-\alpha^{\prime} & \text { if } \beta \geqslant \alpha^{\prime} \\ 2^{\rho}+\beta-\alpha^{\prime} & \text { if } \beta<\alpha^{\prime} .\end{cases}
$$

This number is equal to the number of solutions to Eq. (16) in which $a_{0}$ and $a_{1}$ have the specified values.

We handle case (26) in a way quite similar to (23). We loop over the four possible choices of values for $c_{0}$ and $c_{1}$. For each of these values, we compute the carry $\ell_{2}$ which is added to the right-hand side of $E_{2}$ and let

$$
\beta^{\prime}=4 \ell_{2}+\sum_{i=2}^{\rho-1} \beta_{i} 2^{i}
$$

and we apply the AB Algorithm to count the number of solutions to

$$
\Theta(a, b)=\Theta(b, c)+d
$$

where

$$
d= \begin{cases}\beta^{\prime}-\alpha & \text { if } \quad \beta^{\prime} \geqslant \alpha \\ 2^{\rho}+\beta^{\prime}-\alpha & \text { if } \quad \beta^{\prime}<\alpha .\end{cases}
$$


This number is equal to the number of solutions to Eq. (16) in which $c_{0}$ and $c_{1}$ have the specified values.

With this generalization we call the algorithm the extended AB Algorithm.

\section{CALCULATING THE NUMBER OF CACHE MISSES}

In this section, we return to the problem of counting cache misses for the matrix multiplication example. Recall that we are analyzing the data layout function defined in terms of an $(m, m)$-interleaving $\sigma=\sigma_{2 m-1} \cdots \sigma_{1} \sigma_{0}$, where $A_{i, k}$ maps to $\mu_{1}+\Theta(i, k), B_{k, j}$ maps to $\mu_{2}+\Theta(k, j)$, and $C_{i, j}$ maps to $\mu_{3}+\Theta(i, j)$. We use the following suggestive notation to classify misses.

- A miss is the number of cache misses when accessing an element of $\mathrm{A}$. The quantities $\mathbf{B}$ miss and $\mathbf{C}$ miss are defined analogously.

- A-B miss is the number of cache misses which occur when an element of A is accessed which was in cache but was removed because an element of $\mathrm{B}$ took its place. The quantities $\mathbf{A}-\mathbf{A}$ miss, $\mathbf{A}-\mathbf{C}$ miss, $\mathbf{B}-\mathbf{A}$ miss, $\mathbf{B}-\mathbf{B}$ miss, $\mathbf{B}-\mathbf{C}$ miss, C-A miss, $\mathbf{C}-\mathbf{B}$ miss, and $\mathbf{C}-\mathbf{C}$ miss are defined analogously. Misses of these kinds correspond to the number of solutions of the problems discussed in Sections 2.1 and 2.2 .

- A-BC miss is the number of cache misses which occur when an element of $\mathrm{A}$ is accessed which was previously in cache and such that both an element of B and an element of $\mathrm{C}$ have taken its place in cache since it was most recently there. Other misses of this nature are defined analogously. These correspond to the joint solutions of the $A B$ and $A C$ problems, as discussed in Section 2.3. Recall that we proved there that the number of such joint solutions is very small.

Considering the inclusion-exclusion property of set intersections, the task is to enumerate the following types of misses:

$$
\begin{aligned}
& \mathbf{A} \text { miss }=\mathbf{A}-\mathbf{A} \text { miss }+\mathbf{A}-\mathbf{B} \text { miss }+\mathbf{A}-\mathbf{C} \text { miss }-\mathbf{A}-\mathbf{A B} \text { miss } \\
& \text { - A-BC miss - A-AC miss + A-ABC miss } \\
& \mathbf{B} \text { miss }=\mathbf{B}-\mathbf{A} \text { miss }+\mathbf{B}-\mathbf{B} \text { miss }+\mathbf{B}-\mathbf{C} \text { miss }-\mathbf{B}-\mathbf{A B} \text { miss } \\
& -\mathrm{B}-\mathrm{BC} \text { miss }-\mathrm{B}-\mathrm{AC} \text { miss }+\mathrm{B}-\mathrm{ABC} \text { miss } \\
& \mathrm{C} \text { miss }=\mathrm{C}-\mathrm{A} \text { miss }+\mathrm{C}-\mathrm{B} \text { miss }+\mathrm{C}-\mathrm{C} \text { miss }-\mathrm{C}-\mathbf{A B} \text { miss } \\
& \text { - C-BC miss }-\mathrm{C}-\mathrm{AC} \text { miss }+\mathrm{C}-\mathrm{ABC} \text { miss }
\end{aligned}
$$

Figure 1 shows this for A miss.

However in the special case of matrix multiplication, some misses need not be considered; in particular there are no $\mathbf{A}-\mathbf{A}, \mathbf{A}-\mathbf{A B}, \mathbf{A}-\mathbf{A C}$, or $\mathbf{A}-\mathbf{A B C}$ misses because unique elements $A_{i, k}$ are accessed in the two outermost loops only. A method to derive the types of misses that are required in the more general case of programs other than matrix multiplication is subject of future work. 


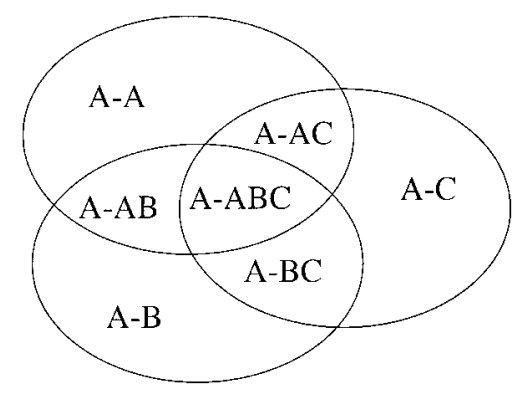

FIG. 1. A miss: inclusion-exclusion property.

Note that we are not including every type of miss in our analysis, but are including a case that represents each of the key ideas involved in counting the number of cache misses.

Throughout this section, we will continue to assume that $2 m \leqslant \rho$ to simplify the exposition. In the case that $2 m>\rho$, the following changes must be made to the analysis in this section. Each time an iteration point $(i, k, j)$ is counted as a miss, then only initial segments of the binary expansions of $i, k$, and $j$ are determined.

There are no constraints on how these initial segments are extended to give complete binary expansions of $i, k$, and $j$. So each miss enumerated in this section must be multiplied by $2^{D}$, where $D$ is the total number of undetermined binary digits in $i, k$ and $j$ (the number $D$ depends on which kind of miss is being enumerated and so must be determined on a case by case basis).

\subsection{Computing A miss}

In this subsection we show how to efficiently compute A miss. An array element $A_{i, k}$ will be accessed at the $n$ iteration points $(i, k, w)$, where $0 \leqslant w \leqslant n-1$. Suppose that we have a cache miss when $A_{i, k}$ is accessed at the iteration point $(i, k, j)$. As the same element of $A$ is accessed throughout the innermost loop, there are no $\mathbf{A}-\mathbf{A}$ misses. Since we are using the lexicographic ordering $i \succ k \succ j$, the iteration point $(i, k, j)$ is immediately preceded by the iteration point $(i, k, j-1)$ at which the array element $A_{i, k}$ is accessed. Thus at the iteration point $(i, k, j-1)$ there must be a memory access of an element of $B$ or $C$ which occupies the same cache set as $A_{i, k}$.

Although possibly negligible, there could also be a small number of contributions to A miss along the boundary of the innermost loop. The array element $A_{i, k-1}$ is accessed during the $(i, k-1, n-1)$ iteration step and the array element $A_{i, k}$ is accessed during the following iteration step, $(i, k, 0)$. Suppose $A_{i, k-1}$ and $A_{i, k}$ occupy the same cache word, then a cache miss occurs if there is a memory access of an element of $B$ or $C$ that maps to the same cache set as $A_{i, k-1}$ at $(i, k-1$, $n-1)$. It will be the case that the array element $A_{i, k}$ existed in the cache, but was removed by an access to $B_{k-1, n-1}$ or $C_{i, n-1}$ at iteration step $(i, k-1, n-1)$.

We can now examine $\mathbf{A}-\mathbf{B}$ miss and $\mathbf{A}-\mathbf{C}$ miss separately. 


\subsubsection{Computing A-B miss}

During the $(i, k, j)$ iteration step we form the product $A_{i, k} \cdot B_{k, j}$ and add it to $C_{i, j}$. When we do so, we access these three pieces of information in the order $A_{i, k}$ followed by $B_{k, j}$ followed by $C_{i, j}$. So, in order for this cache miss to contribute to A-B miss, it must be the case that the array element $A_{i, k}$ was removed from cache at the previous iteration step when the array element $B_{k, j-1}$ was accessed, i.e., $A_{i, k}$ and $B_{k, j-1}$ occupy the same word in cache. This is equivalent to

$$
\left\lfloor\frac{\mu_{1}+\Theta(i, k)}{4}\right\rfloor=\left\lfloor\frac{\mu_{2}+\Theta(k, j-1)}{4}\right\rfloor \bmod 2^{\rho-2},
$$

where this equation is taken $\bmod \frac{p}{4}=2^{\rho-2}$. So $\mathbf{A}-\mathbf{B}$ miss is equal to the number of solutions $(i, k, j)$ to Eq. (27) with $0 \leqslant i \leqslant n-1,0 \leqslant k \leqslant n-1$ and $1 \leqslant j \leqslant n-1$. The number of solutions to Eq. (27) is computed by the Extended AB Algorithm.

To count A-B misses along the boundary of the innermost loop, we determine if $A_{i, k-1}$ and $A_{i, k}$ occupy the same cache word

$$
\left\lfloor\frac{\mu_{1}+\Theta(i, k-1)}{4}\right\rfloor=\left\lfloor\frac{\mu_{1}+\Theta(i, k)}{4}\right\rfloor
$$

and if so, we check if an access to the array element $B_{k-1, n-1}$ causes a cache miss

$$
\left\lfloor\frac{\mu_{1}+\Theta(i, k-1)}{4}\right\rfloor=\left\lfloor\frac{\mu_{2}+\Theta(k-1, n-1)}{4}\right\rfloor \bmod 2^{\rho-2}
$$

incrementing the A-B miss count if both equations are satisfied.

\subsubsection{Computing A-C miss}

By the same reasoning as above, the number of cache misses that contribute to A-C miss is the number of solutions to

$$
\left\lfloor\frac{\mu_{1}+\Theta(i, k)}{4}\right\rfloor=\left\lfloor\frac{\mu_{3}+\Theta(i, j-1)}{4}\right\rfloor \bmod 2^{\rho-2},
$$

where this equation is taken modulo $\frac{p}{4}=2^{\rho-2}$ and $i, j, k$ are constrained to lie in the intervals $0 \leqslant i \leqslant n-1,0 \leqslant k \leqslant n-1$ and $1 \leqslant j \leqslant n-1$. The number of solutions to Eq. (29) is computed by the Extended AC Algorithm.

To count the contributions to A-C miss along the boundary of the innermost loop, we check if $A_{i, k-1}$ and $A_{i, k}$ occupy the same cache word exactly as in Eq. (28), and if so we determine if an access to the array element $C_{i, n-1}$ causes a cache miss

$$
\left\lfloor\frac{\mu_{1}+\Theta(i, k-1)}{4}\right\rfloor=\left\lfloor\frac{\mu_{3}+\Theta(i, n-1)}{4}\right\rfloor \bmod 2^{\rho-2}
$$

incrementing the A-C miss count if both equations are satisfied. 


\subsubsection{Computing A-BC miss}

We will count zero A-BC misses. The conditions in Section 2.3 can be checked in $O(\rho)$ steps to determine whether this count is accurate. As proved in Section 2.3, even if there are instances of such misses, their number is small-less than $2^{-T}$ of the total number of misses, where $T$ is the number of ones in the set $\left\{\sigma_{2}, \sigma_{3}, \ldots, \sigma_{\rho-1}\right\}$. In fact, on the basis of this result, we are setting all terms requiring the simultaneous solving of equations (e.g., B-AB miss, B-BC miss, B-AC miss, B-ABC miss, C-AB miss, C-BC miss, C-AC miss, $\mathrm{C}-\mathbf{A B C}$ miss) to zero.

\subsection{Computing $\mathrm{C}$ miss}

The quantity $\mathbf{C}$ miss counts the number of iteration points $(i, k, j)$ with $k>0$ such that the matrix element $C[i, k, j]$ is not in cache thereby causing a miss. As a first step, we will determine $L[i, k, j]$ which denotes the most recent iteration step, prior to $(i, k, j)$ at which $C[i, k, j]$ was in cache. Note that $L[i, k, j]$ is the most recent iteration step when an element of $C$ was accessed that occupies the same cache word as $C[i, k, j]$. If we write $L[i, k, j]=\left(i^{\prime}, k^{\prime}, j^{\prime}\right)$ this is equivalent to

$$
\left\lfloor\frac{\mu_{3}+\Theta(i, j)}{4}\right\rfloor=\left\lfloor\frac{\mu_{3}+\Theta\left(i^{\prime}, j^{\prime}\right)}{4}\right\rfloor .
$$

\subsubsection{Computing $\mathbf{C}-\mathbf{A}$ miss}

The solution to Eq. (30) depends on the form of $\Theta$ and so at this point the analysis must break into cases. There are four cases to consider depending on whether $\sigma_{1} \sigma_{0}=00,01,10$ or 11 . We will write out details in two of the cases which represent the technical problems that come up in the other two cases. The details of the remaining two cases are left to the reader.

Case 1. $\sigma_{1} \sigma_{0}=00$.

In this case, the four elements of $C$ which occupy the same cache word as $C[i, k, j]=C_{i, j}$ are usually $C_{i, j-u}, C_{i, j-u+1}, C_{i j-u+2}, C_{i, j-u+3}$, where $\mu_{3}+$ $\Theta(i, j) \equiv u \bmod 4$. The modifier "usually" refers to the observation that not all of these elements of $C$ might exist in the extreme cases where $j<u$ or $j-u+3 \geqslant n$. But as long as $u>0$ and $j>0, C_{i, j-1}$ is in the same cache word as $C_{i, j}$. In this case, $C[i, k, j]$ is brought into cache at the preceding iteration step $(i, k, j-1)$ and so $L(i, k, j)=(i, k, j-1)$.

If $j=0$ or $u=0$ then $L(i, k, j)=(i, k-1, j-u+3)$ unless $j-u+3 \geqslant n$. In that case $(u=0$ and $j-u+3 \geqslant n), L(i, k, j)=(i, k-1, n-1)$. To summarize: if $u \equiv$ $\mu_{3}+\Theta(i, j) \bmod 4$, then

$$
L(i, k, j)=\left\{\begin{array}{l}
(i, k, j-1) \text { if } j>0 \text { and } u>0 \\
(i, k-1, j-u+3) \text { if } j=0 \text { or }\{u=0 \text { and } j-u+3<n\} \\
(i, k-1, n-1) \text { if } u=0 \text { and } j-u+3 \geqslant n .
\end{array}\right.
$$


Now $\mathbf{C}-\mathbf{A}$ miss is the number of pairs of iteration points $(i, k, j),(x, z, y)$ such that

$$
L(i, k, j)<(x, z, y) \leqslant(i, k, j)
$$

and

$$
\left\lfloor\frac{\mu_{1}+\Theta(x, z)}{4}\right\rfloor=\left\lfloor\frac{\mu_{3}+\Theta(i, j)}{4}\right\rfloor \bmod 2^{\rho-2} .
$$

To clarify the connection between $\mathbf{C}-\mathbf{A}$ miss, Eq. (31), and Eq. (32) note that Eq. (32) states that $A[x, z, y]$ and $C[i, k, j]$ occupy the same cache word and Eq. (31) states that iteration step $(x, z, y)$ occurs sometime between the iteration step $(i, k, j)$ and then previous iteration step when $C[i, k, j]$ was brought into cache.

We now break our analysis into two cases depending on the exact form of $L[i, k, j]$. If $L[i, k, j]=(i, k, j-1)$ then we must have $(x, z, y)=(i, k, j)$. Also, if $u=0$ and $j+3 \geqslant n$ so that $L[i, k, j]=(i, k-1, n-1)$ then Eq. (31) becomes $(i, k-1, n-1)<(x, z, y) \leqslant(i, k, j)$. This cannot be satisfied with $z=k-1$ because we would then need $n-1<y$. So we must have $z=k$ and $y=j$. This is a second instance in which $(x, z, y)$ must be equal to $(i, k, j)$. In this case Eq. (32) states

$$
\left\lfloor\frac{\mu_{1}+\Theta(i, k)}{4}\right\rfloor=\left\lfloor\frac{\mu_{3}+\Theta(i, j)}{4}\right\rfloor \bmod 2^{\rho-2} .
$$

Solutions to this equation are enumerated by the Extended AC Algorithm.

If $j=0$ or $u=0$ and $j-u+3 \leqslant n-1$ then Eq. (31) states that $(i, k-1, j-u+3)$ $<(x, z, y) \leqslant(i, k, j)$. We deduce that $x=i$ and that $z$ is equal to either $k-1$ or $k$. Also, in this case we cannot satisfy the inequality $j-u+3<y \leqslant j$ so we must have $z=k-1$. Thus the contribution to $\mathbf{C}-\mathbf{A}$ miss made in this case is number of solutions to Eq. (32) which is

$$
\left\lfloor\frac{\mu_{1}+\Theta(i, k-1)}{4}\right\rfloor=\left\lfloor\frac{\mu_{3}+\Theta(i, j)}{4}\right\rfloor \bmod 2^{\rho-2} .
$$

This is equivalent to enumerating solutions to

$$
\left\lfloor\frac{\mu_{1}+\Theta\left(i, k^{\prime}\right)}{4}\right\rfloor=\left\lfloor\frac{\mu_{3}+\Theta(i, j)}{4}\right\rfloor \bmod 2^{\rho-2},
$$

where $0 \leqslant i \leqslant n-1,0 \leqslant k^{\prime} \leqslant n-2,0 \leqslant j \leqslant n-1$. Solutions to this equation are enumerated by the Extended AC algorithm.

This completes Case 1 in our analysis of $\mathbf{C}-\mathbf{A}$ miss. 
Case 2. $\sigma_{1} \sigma_{0}=10$.

The fundamental difference between the analysis in this case and the analysis in Case 1 is the relationship between cache words and the arrays $A, B, C$. In particular, the elements of $C$ that occupy the same cache word as $C_{i, j}$ are

$$
C_{i-v, j-u}, C_{i-v+1, j-u}, C_{i-v, j-u+1}, C_{i-v+1, j-u+1},
$$

where

$$
\mu_{3}+\Theta(i, j) \equiv v \bmod 2
$$

and

$$
\frac{\mu_{3}+\Theta(i, j)-v}{2} \equiv u \bmod 2
$$

The analysis now parallels the analysis in Case 1 but with changes in some details to reflect the cache word structure given in Eq. (33).

If $j>0$ and $u>0$ the $L[i, k, j]=(i, k, j-1)$ and we proceed as in Case 1. If $u=0$ and $j=n-1$ then $L[i, k, j]=(i, k-1, n-1)=(i, k-1, j)$. In both these cases, if a cache miss is caused by the access of $A_{x, z}$ removing $C_{i, j}$ at iteration step $(x, z, y)$, where $L[i, k, j]<(x, z, y) \leqslant(i, k, j)$, then we must have $(x, z, y)=(i, k, j)$. These instances are enumerated as in Case 1 by the Extended AC algorithm.

If $j=0$ or if $u=0$ and $j<n-1$ then $L(i, k, j)=(i, k-1, j-a+1)$. In this case, $(x, z, y)$ must equal $(i, k-1, j-u+1)$ and we enumerate these instances as in Case 1. This completes the computation of $\mathbf{C}-\mathbf{A}$ miss in Case 2.

The computation of $\mathbf{C}-\mathbf{A}$ miss in the remaining two cases is similar.

Note that $\mathbf{C}-\mathbf{C}$ miss can be handled in a manner similar to $\mathbf{C}-\mathbf{A}$ miss. There is the same consideration of the most recent iteration step at which an element of $C$ was addessed that occupies the same cache word as $C[i, k, j]$, and the analysis breaks into the same four cases depending on $\sigma_{1} \sigma_{0}$. The key difference is that in this case, an access to $C_{x, y}$ interferes with an access to $C_{i, j}$, where $x, y$ are as in Eq. (31).

\subsubsection{Computing $\mathbf{C}-\mathbf{B}$ miss}

To compute $\mathbf{C}-\mathbf{B}$ miss, we need to compute the number of pairs of triples $(i, k, j),(x, z, y)$ which satisfy Eq. (31) such that $C[i, k, j]=C_{i, j}$ and $B[x, z, y]$ $=B_{z, y}$ occupy the same cache block. This latter condition is equivalent to

$$
\left\lfloor\frac{\mu_{3}+\Theta(i, j)}{4}\right\rfloor=\left\lfloor\frac{\mu_{2}+\Theta(z, y)}{4}\right\rfloor \bmod 2^{\rho-2} .
$$


As in the previous subsection, if

$$
\left\lfloor\frac{\mu_{3}+\Theta(i, j-1)}{4}\right\rfloor=\left\lfloor\frac{\mu_{3}+\Theta(i, j)}{4}\right\rfloor,
$$

then Eq. (31) implies that $(x, z, y)=(i, k, j)$. In that case, Eq. (34) is equivalent to

$$
\left\lfloor\frac{\mu_{3}+\Theta(i, j)}{4}\right\rfloor=\left\lfloor\frac{\mu_{2}+\Theta(k, j)}{4}\right\rfloor \bmod 2^{\rho-2} .
$$

Let $\hat{\sigma}$ be the interleaving obtained from $\sigma$ by interchanging 0 's and 1's and let $\hat{\Theta}$ denote the mixing function determined by $\hat{\sigma}$. Note that for any pair of nonnegative integers $v, w$ :

$$
\Theta(v, w)=\hat{\Theta}(w, v)
$$

Thus, we can rewrite Eq. (35) by

$$
\left\lfloor\frac{\mu_{3}+\hat{\Theta}(j, i)}{4}\right\rfloor=\left\lfloor\frac{\mu_{2}+\hat{\Theta}(j, k)}{4}\right\rfloor \bmod 2^{\rho-2} .
$$

The Extended AC Algorithm counts solutions to Eq. (36) which gives us a fast algorithm to count contributions to $\mathbf{C}-\mathbf{B}$ miss that arise in the instances where $(x, z, y)=(i, k, j)$.

The remaining contributions to $\mathbf{C}-\mathbf{B}$ miss come from solutions to Eq. (34) in the cases where either $j=0$ or

$$
\left\lfloor\frac{\mu_{3}+\Theta(i, j-1)}{4}\right\rfloor \neq\left\lfloor\frac{\mu_{3}+\Theta(i, j)}{4}\right\rfloor .
$$

The analyses of these two cases are somewhat different and so we do them separately.

Consider the case where

$$
\left\lfloor\frac{\mu_{3}+\Theta(i, j-1)}{4}\right\rfloor \neq\left\lfloor\frac{\mu_{3}+\Theta(i, j)}{4}\right\rfloor .
$$

This can occur in one of three different ways. If $\sigma_{0}=1$ then this condition is equivalent to $\mu_{3}+\Theta(i, j) \equiv 0 \bmod 4$. If $\sigma_{1} \sigma_{0}=10$ then this condition is equivalent to $\mu_{3}+\Theta(i, j) \equiv 0,1 \bmod 4$. Finally, if $\sigma_{1} \sigma_{0}=00$ then this condition always holds.

In this case, we have $L(i, k, j)=(i, k-1, j+1)$ and so Eq. (31) becomes

$$
(i, k-1, j+1)<(x, z, y) \leqslant(i, k, j) .
$$

At first glance, the enumeration of solutions to Eq. (34) appears to be problematic. Although we can deduce that $z$ is either $k-1$ or $k$, we have very little control on $j$. 
So Eq. (34) contains four variables that are essentially independent. After some simplification of the problem, we will see that this is in fact an advantage and makes the enumeration of solutions particularly easy.

To count solutions to Eq. (34) we first loop over all possible values for the first two digits in the binary expansions of $\Theta(i, j)$ and $\Theta(z, y)$. This will involve specifying the first two digits of $i$, or the first digit of $i$ and the first digit of $j$, or the first two digits of $j$ depending on the values of $\sigma_{1} \sigma_{0}$. Let $i^{\prime}, j^{\prime}$ be the remaining, unspecified digits of $i$ and $j$. Define $z^{\prime}$ and $y^{\prime}$ similarly. Also, define $\Theta^{\prime}$ to be the mixing function associated with $\sigma^{\prime}=\cdots \sigma_{3} \sigma_{2}$.

Having specified the first two digits of $\Theta(i, j)$ and $\Theta(z, y)$ we next perform the following steps:

1. Check whether

$$
\left\lfloor\frac{\mu_{3}+\Theta(i, j-1)}{4}\right\rfloor=\left\lfloor\frac{\mu_{3}+\Theta(i, j)}{4}\right\rfloor .
$$

If so, go to the next step in the loop (here loop refers to the outermost loop whose steps are indexed by the choices for possible first two digits of $\Theta(i, j)$ and $\Theta(z, y)$ ). Otherwise, continue to step 2).

2. Let $\mu_{3}^{\prime}$ be $\left\lfloor\frac{\mu_{3}}{4}\right\rfloor+\epsilon_{1}$ where $\epsilon_{1}$ is the binary carry from the first to the second binary digits in $\mu_{3}+\Theta(i, j)$.

3. Let $\mu_{2}^{\prime}$ be $\left\lfloor\frac{\mu_{2}}{4}\right\rfloor+\tau_{1}$ where $\tau_{1}$ is the binary carry from the first to the second binary digits in $\mu_{2}+\Theta(z, y)$.

The number of solutions to Eq. (34) given the specified digits in $\Theta(i, j)$ and $\Theta(z, y)$ is equal to the number of solutions to

$$
\mu_{3}^{\prime}+\Theta^{\prime}\left(i^{\prime}, j^{\prime}\right)=\mu_{2}^{\prime}+\Theta^{\prime}\left(z^{\prime}, y^{\prime}\right) \bmod 2^{\rho-2} .
$$

Define $d$ by

$$
d= \begin{cases}\mu_{3}^{\prime}-\mu_{2}^{\prime} & \text { if } \mu_{3}^{\prime} \geqslant \mu_{2}^{\prime} \\ \frac{p}{4}+\mu_{3}^{\prime}-<u_{2}^{\prime} & \text { if } \mu_{3}^{\prime}<\mu_{2}^{\prime} .\end{cases}
$$

Then the number of solutions to Eq. (37) is equal to the number of solutions to Eq. (38):

$$
d+\Theta^{\prime}\left(i^{\prime}, j^{\prime}\right)=\Theta^{\prime}\left(z^{\prime}, y^{\prime}\right) \bmod 2^{\rho-2}
$$

Enumerating solutions to Eq. (38) is straightforward. Let $d^{\prime}$ consist of the first $2 m-2$ binary digits of $d$. First, examine the remaining binary digits, $d_{\ell}$ for $2 m-2 \leqslant \ell \leqslant \rho-3$. Unless these remaining binary digits are identical, there are no solutions to Eq. (38). If they are identical and equal to $\epsilon$, then the number of solutions to Eq. (38) is equal to the number of solutions to

$$
d^{\prime}+\Theta^{\prime}\left(i^{\prime}, j^{\prime}\right)=\Theta^{\prime}\left(z^{\prime}, y^{\prime}\right) \bmod 2^{2 m-2}
$$


for which the terminal carry on the left hand side of Eq. (39) is $\epsilon$. The key observation is that the number of solutions to Eq. (39) is equal to the number of pairs $\left(i^{\prime}, j^{\prime}\right)$ which result in terminal carry $\epsilon$-once such a pair has been specified there is a unique choice of $z^{\prime}, y^{\prime}$ which satisfy Eq. (39).

In our design of the AC Algorithm (Section 2), we exactly determined the number of pairs $i^{\prime}, j^{\prime}$ which give terminal carry $\epsilon$ on the left-hand side of Eq. (39). This number is:

$$
\begin{cases}2^{2 m-2}-d^{\prime} & \text { if } \epsilon=0 \\ d^{\prime} & \text { if } \epsilon=1 .\end{cases}
$$

This finishes our enumeration of solutions to Eq. (34) in the case that

$$
\left\lfloor\frac{\mu_{3}+\Theta(i, j-1)}{4}\right\rfloor \neq\left\lfloor\frac{\mu_{3}+\Theta(i, j)}{4}\right\rfloor .
$$

To evaluate the complexity of this enumeration algorithm: there is an outer loop through the 16 possible choices for the first two binary digits of $\Theta(i, j)$ and $\Theta(z, y)$. Within that loop, we need to determine $d$ and check whether the binary digits of $d$ are consistent between $2 m-2$ and $\rho-3$. That takes $O(\rho)$ operations. Then we need to compute $d^{\prime}$ which can be done in $O(m)$ operations. Thus the total complexity in this case is $O(\rho)$.

To complete the calculation of $\mathbf{C}-\mathbf{B}$ miss we need to consider the case where $j=0$. By the same series of reductions we used in the previous case, this reduces to enumerating solutions to

$$
d^{\prime}+\Theta^{\prime}\left(i^{\prime}, 0\right)=\Theta^{\prime}\left(z^{\prime}, y^{\prime}\right) \bmod 2^{2 m-2} .
$$

As before, Eq. (40) has no solutions unless the binary digits $d_{\ell}, \ell=\rho-3, \ldots, 2 m-1$, $2 m-2$ are identical. If they are identical, let $\epsilon \in\{0,1\}$ represent their common value. In that case, the number of solutions to Eq. (40) is equal to the number of $i^{\prime}$ for which $d^{\prime}+\Theta^{\prime}\left(i^{\prime}, 0\right)$ has terminal carry $\epsilon$.

To efficiently compute this number, first scan the left hand sides of the equations in Eq. (40) from bottom to top. Consider those left-hand sides which have the form $d_{\ell}^{\prime}+0+k_{\ell-1}$ (where 0 comes from the $j^{\prime}=0$ component in $\Theta^{\prime}\left(i^{\prime}, 0\right)$, i.e., $\sigma_{\ell+2}=1$ ). If $d_{\ell}^{\prime}=1$ then erase that equation as you know that whatever carry comes in, the same carry will go out. If $d_{\ell}^{\prime}=0$ then stop your scan. You know that $k_{\ell}$ will have to be 0 . So we can start constructing solutions from the next equation on without regard to any earlier binary digits of $i^{\prime}$. To this end, let $\tau$ be the number of $i_{a}^{\prime}$ which occur in equation in Eq. (40) that comes before your stopping point. Let $d^{\prime \prime}$ be the digits of $d^{\prime}$ that remain on the left-hand side of Eq. (40) above the $\ell$ th digit (we use the word remain because we have erased some equations at earlier-steps in the scan). Let $i^{\prime \prime}$ be the corresponding digits of $i^{\prime}$ and let $\mu$ be the number of digits of $d^{\prime \prime}$. Then the number of solutions to Eq. (40) is $2^{\tau}$ times the number of $i^{\prime \prime}$ such that

$$
\begin{cases}d^{\prime \prime}+i^{\prime \prime}<2^{\mu} & \text { if } \quad \epsilon=0 \\ d^{\prime \prime}+i^{\prime \prime} \geqslant 2^{\mu} & \text { if } \quad \epsilon=1 .\end{cases}
$$


This number is computed as before which completes the $j=0$ case and therefore completes our computation of $\mathbf{C}-\mathbf{B}$ miss.

Note that the algorithm described here to handle the case $j=0$ has complexity $O(\rho)$. Also note that for each choice of initial two digits in $\Theta(i, j)$, the solutions to Eq. (34) where $j=0$ are either contained in, or else disjoint from, the solutions where

$$
\left\lfloor\frac{\mu_{3}+\Theta(i, j-1)}{4}\right\rfloor \neq\left\lfloor\frac{\mu_{3}+\Theta(i, j)}{4}\right\rfloor .
$$

It is trivial to determine whether there is inclusion by examination of the initial two digits chosen which takes care of any overcounting that results from iteration steps $(i, k, j)$ that are enumerated in both of these cases.

\subsection{Computing $B$ miss}

In this subsection we finish the analysis of misses by computing $\mathbf{B}$ miss. The quantity $\mathbf{B}$ miss counts the number of iteration points $(i, k, j)$ at which the matrix element $B_{k, j}$ is not in cache, having been there previously.

If $B_{k, j}$ is in the same cache block as $B_{k, j-1}$, (Note. This case will arise if $\sigma_{0}=1$ and $\mu_{2}+\Theta(k, j) \not \equiv 1,2,3 \bmod 4$, or if $\sigma_{1} \sigma_{0}=10$ and $\mu_{2}+\Theta(k, j) \equiv 2,3 \bmod 4$.) any collisions that forced $B_{k, j}$ out of cache must have occured at iteration step $(i, k, j-1)$ if the collision occurs with an element of $C$ or at step $(i, k, j)$ if the collision occurs with an element of $A$. Using arguments that are similar to those in previous cases, we see that these instances are enumerated by the Extended AB Algorithm and the Extended AC Algorithm. So we only need to examine the cases where $B_{k, j}$ and $B_{k, j-1}$ occupy different cache words. This analysis depends on the form of $\sigma$ and so we need to consider different cases.

\subsubsection{Computing B-A miss}

We want to add one to $\mathbf{B}-\mathbf{A}$ miss if there is an iteration step $(x, z, y)$ with

$$
(i, k-1, j+1)<(x, z, y) \leqslant(i, k, j)
$$

for which the matrix entry $A_{x, z}$ occupies the same cache word as $B_{k, j}$. Note that $x=i$ and that $z=k-1$ or $z=k$. We are free to choose $y$ as long as we choose $y>j+1$ when $z=k$ and $y \leqslant j$ when $z=k$.

Case 1. $B_{k, j}$ is in the same cache word as $B_{k-1, j}$ but not in the same cache word as $B_{k, j-1}$. (Note: This case will arise if $\sigma_{1} \sigma_{0}=10$ and $\mu_{2}+\Theta(k, j) \equiv 1 \bmod 4$ or if $\sigma_{1} \sigma_{0}=00$ and $\left.\mu_{2}+\Theta(k, j) \equiv 1,2,3 \bmod 4\right)$.

In this case, $B_{k, j}$ is brought into cache at iteration step $(i, k, j-1)$. The enumeration of misses in this case is similar to previous cases. 
Enumerating $(i, k, j)$ which satisfy these conditions is equivalent to counting $(i, k, j)$ which are solutions to:

$$
\left\lfloor\frac{\mu_{2}+\Theta(k, j)}{4}\right\rfloor=\left\lfloor\frac{\mu_{1}+\Theta(i, k)}{4}\right\rfloor \bmod 2^{\rho-2}
$$

(we can then choose any $y \leqslant j$ ) OR which are solutions to

$$
\left\lfloor\frac{\mu_{2}+\Theta(k, j)}{4}\right\rfloor=\left\lfloor\frac{\mu_{1}+\Theta(i, k-1)}{4}\right\rfloor \bmod 2^{\rho-2}
$$

with $j<n-1$ (we can then choose any $y>j+1$ ). In doing so, we must be careful to count those $(i, k, j)$ which satisfy both sets of equations only once. Fortunately, because we are in Case 2, we know that

$$
\left\lfloor\frac{\mu_{2}+\Theta(k, j)}{4}\right\rfloor=\left\lfloor\frac{\mu_{2}+\Theta(k-1, j)}{4}\right\rfloor .
$$

So we can replace Eq. (42) with an identical equation which has $k-1$ in place of $k$ on the left hand side. When this is done, Eq. (42) is identical to Eq. (41) with the variable $k^{\prime}=k-1$ in place of $k$. So, to count $(i, k, j)$ which are solutions to at least one of Eq. (41) or Eq. (42), we can just count solutions to Eq. (41). The number of solutions to Eq. (41) can be computed as in previous cases.

Case 2. $B_{k, j}$ is in a different cache block from both $B_{k-1, j}$ and $B_{k-1, j}$. (Note: This arises exactly when $\left.\mu_{2}+\Theta(k, j) \equiv 0 \bmod 4\right)$.

In this case, the most recent previous access of $B_{k, j}$ was at iteration step

$$
\begin{array}{rlll}
(i-1, k+3, j) & \text { if } & \sigma_{1} \sigma_{0}=00 & \\
(i-1, k+1, j+3) & \text { if } & \sigma_{1} \sigma_{0}=01 \quad \text { or } 10 \\
(i-1, k, j+3) & \text { if } & \sigma_{1} \sigma_{0}=11 . &
\end{array}
$$

We will consider just one of these possibilities - the others are handled in similar ways. Assume that $\sigma_{1} \sigma_{0}=10$.

The iteration step $(i, k, j)$ contributes to $\mathbf{B}-\mathbf{A}$ miss if there is an iteration step $(x, z, y)$ with

$$
(i-1, k+1, j+1)<(x, z, y) \leqslant(i, k, j)
$$

satisfying

$$
\left\lfloor\frac{\mu_{3}+\Theta(k, j)}{4}\right\rfloor=\left\lfloor\frac{\mu_{1}+\Theta(x, z)}{4}\right\rfloor \bmod 2^{\rho-2}
$$

This is similar to the exceptional case for $\mathbf{C}-\mathbf{B}$ miss, where $\mu_{3}+\Theta(i, j) \equiv 0 \bmod 4$. We enumerate solutions in a similar way. 
Note that $\mathbf{B}-\mathbf{B}$ miss and $\mathbf{B}-\mathbf{C}$ miss are computed in ways quite similar to $\mathbf{B}-\mathbf{A}$ miss, dividing the analysis in the same Case 1 and Case 2. For $\mathbf{B}-\mathbf{B}$ miss, we are counting triples $(i, k, j)$ such that the array element $B_{k, j}$ was in cache but was removed because array element $B_{z, y}$ took its place in cache. For B-C miss, we are counting triples $(i, k, j)$ such that $B_{k, j}$ was displaced from cache by $C_{x, y}$.

This completes the enumeration of cache misses.

\section{A-WAY ASSOCIATIVE CACHE}

In this section, we indicate the changes needed to generalize our enumeration of cache misses from direct mapped cache to the case of an $\mathbb{A}$-way associative cache (Fig. 2). In this case, memory location $M$ is mapped to the cache set $\Lambda=\left\lfloor\frac{M}{4}\right\rfloor \bmod p_{\mathrm{A}}$, where $p_{\mathbb{A}}=\frac{p}{\mathbb{A}}$ is the number of cache sets. $\Lambda$ contains $\mathbb{A}$ cache blocks (each consisting of four memory locations, as explained in Section 3 ) that are filled according to either the first-in, first-out (FIFO) protocol, the least recently used (LRU) protocol or random fill [6]. LRU gives the best performance but is usually the most difficult to describe. We will show the analysis given the LRU protocol.

Assuming the LRU protocol, a cache block is evicted on a cache miss when its last access lies furthest back in time, i.e., in our framework, we must enumerate instances where a matrix element $X$ is accessed and brought into the cache set $\Lambda$, and where at least $\mathbb{A}$ times, since the previous access of $X$, different matrix elements are accessed that are not in cache and which are mapped to the same cache set $\Lambda$. We will use the term collisions for such instances and call these instances collisions with $X$. For more specificity, we will characterize collisions according to what kinds of array elements are involved. So, we will talk about $\mathbf{C}-\mathbf{A}$ collisions meaning instances when an array element from $A$ is brought into the same cache set as an element of $C$ between consecutive accesses of that element of $C$. The relationship between collisions and cache misses is straightforward-when we access a matrix element $X$, we will have a cache miss if there have been greater than $\mathbb{A}$ collisions with $X$ since the previous access. Thus, misses constitute a subset of collisions.

In the following, we will show the analysis for $\mathbf{C}$ collisions. According to our strategy, we will enumerate iteration steps $(i, k, j)$ according to the number of collisions of type $\mathbf{C}-\mathbf{A}, \mathbf{C}-\mathbf{B}, \mathbf{C}-\mathbf{C}, \mathbf{C}-\mathbf{A B}, \mathbf{C}-\mathbf{A C}, \mathbf{C}-\mathbf{B C}$, and $\mathbf{C}-\mathbf{A B C}$ that have occurred between the access of $C_{i, j}$ at iterations step $(i, k-1, j+\tau)$ and $(i, k, j)$.

The considerations that go into enumeration of collisions will be very similar the considerations that went into the enumeration of cache misses in the direct mapped case, but the general enumeration framework will be somewhat more challenging.

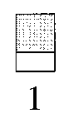

(a)

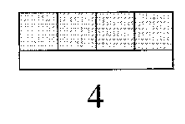

(b)

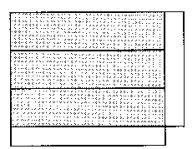

4

(c)
A

FIG. 2. (a) cache word (b) cache block, size 4 (c) cache set, $\mathbb{A}=3$. 
Instead of dividing the analysis according to which matrix is being accessed, we will divide the analysis according to the number of iteration steps since the most recent access of the matrix element under consideration.

Consider the situation where we access a matrix element $X$ at iteration step $(i, k, j)$. At this point, we will not yet specify which of the arrays $A, B$ or $C$ that $X$ comes from.

Case 6.1. The matrix element $X$ was last accessed at iteration step $(i, k, j-1)$.

In this case, the access of $X$ at iteration step $(i, k, j)$ can only cause a cache miss for $L=1,2$. This case can be handled using arguments from the previous section. Note that this case includes all $\mathbf{A}$ misses and a subset of the $\mathbf{C}$ misses.

Case 6.2. The most recent access of $X$ (prior to iteration step $(i, k, j)$ ) was at iteration step $(i, k-1, j+\tau)$ for some $\tau$.

At this point, it is necessary to consider which of the arrays $X$ comes from.

Consider the problem of determining whether there is a cache miss with an A-way associative cache when $C_{i, j}$ is accessed at iteration step $(i, k, j)$. For the next few paragraphs, it is important to keep in mind that $i, k, j$ are fixed. We are going to try to find conditions on $i, k, j$ under which there will be at least $\mathbb{A}$ collisions with $C_{i, j}$ between iteration steps $(i, k-1, j+\tau)$ and $(i, k, j)$.

For $\mathbf{C}-\mathbf{A}$ collisions, let $\alpha$ be the number of distinct $A_{x, z}$ which occupy the same cache set as $C_{i, j}$ and which are accessed between steps $(i, k-1, j+\tau)$ and $(i, k, j)$. By that latter condition, we must have $x=i$ and $z \in\{k-1, k\}$. So, $\alpha=0,1,2$ depending on whether neither, one of, or both of $u=k-1$ and $u=k$ give solutions to

$$
\left\lfloor\frac{\mu_{3}+\Theta(i, j)}{4}\right\rfloor=\left\lfloor\frac{\mu_{1}+\Theta(i, u)}{4}\right\rfloor \bmod 2^{\rho} .
$$

For $\mathbf{C}-\mathbf{B}$ collisions, let $\beta$ be the number of distinct $B_{z, y}$ which occupy the same cache set as $C_{i, j}$ and which are accessed between steps $(i, k-1, j+\tau)$ and $(i, k, j)$. By that latter condition, we must have $x=i$ and $z \in\{k-1, k\}$. To occupy the same cache set as $C_{i, j}$ we must have

$$
\left\lfloor\frac{\mu_{3}+\Theta(i, j)}{4}\right\rfloor=\left\lfloor\frac{\mu_{2}+\Theta(z, y)}{4}\right\rfloor \bmod 2^{\rho} .
$$

Finally for $\mathbf{C}-\mathbf{C}$ collisions, let $\gamma$ be the number of distinct $C_{x, y}$ which occupy the same cache set as $C_{i, j}$ and which are accessed between steps $(i, k-1, j+\tau)$ and $(i, k, j)$. By the latter condition, we must have $x=i$. To occupy the same cache set as $C_{i, j}$ we must have

$$
\left\lfloor\frac{\mu_{3}+\Theta(i, j)}{4}\right\rfloor=\left\lfloor\frac{\mu_{3}+\Theta(x, y)}{4}\right\rfloor \bmod 2^{\rho} .
$$


Since $x=i$, Eq. (44) is equivalent to

$$
\left\lfloor\frac{\mu_{3}+\Theta(i, j)}{4}\right\rfloor=\left\lfloor\frac{\mu_{3}+\Theta(i, y)}{4}\right\rfloor \bmod 2^{\rho} .
$$

Before diving into details, it is worth discussing the broad outlines of the enumeration method that we follow. Our immediate goal is to enumerate $\mathbf{C}$ misses with an $\mathbb{A}$-way associative cache. More precisely, we want to count iteration steps $(i, k, j)$ where the most recent prior access of $C_{i, j}$ was at iteration step $(i, k-1$, $j+\tau)$ and where there have been at least $\mathbb{A}$ distinct matrix elements $X$ inserted into the same cache set as $C_{i, j}$ between iteration steps $(i, k-1, j+\tau)$ and $(i, k, j)$.

The solutions to Eq. (43) characterize those $X=A_{i, u}$ which collide with $C_{i, j}$, the solutions to Eq. (44) characterize those $B_{z, y}$ which collide with $C_{i, j}$ and the solutions to Eq. (46) characterize those $C_{i, y}$ which collide with $C_{i, j}$, all collisions occuring between iteration steps $(i, k-1, j+\tau)$ and $(i, k, j)$.

For any fixed $(i, k, j)$ there can be at most two $\mathbf{C}-\mathbf{A}$ collisions because solutions to Eq. (43) determine $(i, k, j)$. The collisions will occur when $A_{i, u}$ is inserted into the same cache set as $C_{i, j}$. Since $u$ must be either $k$ or $k-1$, there can be at most two such collisions. There are two collisions if $(i, k-1, j)$ and $(i, k, j)$ are simultaneously solutions to Eq. (43) and so we will have to enumerate such instances.

The treatment of $\mathbf{C}-\mathbf{B}$ collisions is more complicated. The number of collisions for a fixed $(i, k, j)$ is the number of solutions to Eq. (44) with $z=k$ and $y \leqslant j$ plus the number of solutions to Eq. (44) with $z=(k-1)$ and $y>j$. If $2 m \leqslant \rho$ then $z$ and $y$ are completely determined by Eq. (44) and so the total number of $\mathbf{C}-\mathbf{B}$ collisions for a fixed $(i, k, j)$ will be at most two. However, if $2 m>\rho$ then you must consider the number of ways you can extend $\bmod 2^{\rho}$ solutions $i, j, z, y$ of Eq. (44). You have unrestricted choice of extensions for $i, z$ thus creating $2^{2 \cdot E_{0}}$ distinct choices for $i, k$. For each choice of extension of $j$ you must count to extensions of $y$ so that $y \leqslant j$ if $z=k$ or $y>j$ if $z=(k-1)$. The number of such extensions of $y$ will dictate the number of $\mathbf{C}-\mathbf{B}$ collisions for this particular $(i, k, j)$. A crucial consideration is whether $(i, k, j)$ and $(i, k-1, j)$ are simultaneously solutions to Eq. (44). If so, any extension of $y$ will create a $\mathbf{C}-\mathbf{B}$ collision without any consideration of how $y$ compares to $j$. So, we will need an algorithm to determine the number of iteration steps $(i, k, j)$ for which there are simultaneous solutions to Eq. (44) with $z=k$ and $z=k-1$.

Considerations of extensions also come into play when counting $\mathbf{C}-\mathbf{C}$ misses. In this case, $k$ is arbitrary so whatever enumeration of collisions we do for a fixed $i$, $j$ will hold for all iteration steps of the form $(i, k, j)$. Again, Eq. (46) determines $y$ (in terms of $i, j) \bmod 2^{\rho}$. We can then extend $i, j, y$ without restriction in digits $\rho$ to $2 m-1$. Different extensions of $i, j$ give different iteration steps $(i, k, j)$ (again, $k$ is free to take on any value). However, different extensions of $y$ give multiple $\mathbf{C}-\mathbf{C}$ collisions at the iteration $\operatorname{step}(i, k, j)$.

This gives a framework for the enumeration. The method will utilize the technology we have already developed, with a couple of simple extensions, to enumerate solutions to Eq. (43), (44), and (46). If $2 m \leqslant \rho$ then this analysis follows closely the analysis of cache misses in the direct mapped case done in Section 4. 
So we will focus on the case where $2 m>\rho$ where there are considerations not previously encountered. In this case, we must consider extensions of solutions to binary digits $\rho$ and beyond. These extensions sometimes expand the number of iteration points $(i, k, j)$ and sometimes expand the number of collisions per iteration point.

When this analysis is complete, we will have counted $\mathbf{C}-\mathbf{A}, \mathbf{C}-\mathbf{B}$ and $\mathbf{C}-\mathbf{C}$ collisions separately. We must then indicate how to count iteration points where there are simultaneous $\mathbf{C}-\mathbf{A}, \mathbf{C}-\mathbf{B}$ and $\mathbf{C}-\mathbf{C}$ collisions. We begin with two technical lemmas that will be key to our analysis.

LEMma 5.1. There is an algorithm, ALGORITHM D1, that counts the number of triples $(i, z, j)$ such that

$$
\left\lfloor\frac{\mu_{3}+\Theta(i, j)}{4}\right\rfloor=\left\lfloor\frac{\mu_{1}+\Theta(i, z)}{4}\right\rfloor=\left\lfloor\frac{\mu_{1}+\Theta(i, z+1)}{4}\right\rfloor \bmod 2^{\rho} .
$$

Moreover, this algorithm has the same complexity as the AC ALGORITHM.

The algorithm proceeds loops over possible first two digits of $\Theta(i, j)$ and $\Theta(i, z)$. For each such choice, the algorithm computes if there is a contribution to the total count and proceeds to the next step in the loop. The complete proof is shown in Appendix A.1.

There is a second situation, similar in nature, in which we will need to count instances where two solutions differ by just one in one of the variables.

LEMMA 5.2. There is an algorithm, ALGORITHM D2, which counts the number of triples $(i, k, j) \in B_{m}^{3}$ such that there are simultaneous solutions $v, x \in B_{m}$ to

$$
\left\lfloor\frac{\mu_{3}+\Theta(i, j)}{4}\right\rfloor=\left\lfloor\frac{\mu_{2}+\Theta(k, v)}{4}\right\rfloor=\left\lfloor\frac{\mu_{2}+\Theta(k-1, x)}{4}\right\rfloor .
$$

In addition, this algorithm will determine the number of solutions to Eq. (48) which satisfy $v \leqslant j<x$. The complexity of this algorithm is $O(\rho)$.

The complete proof is somewhat lengthy. It can be found in Appendix A.2.

We are now ready to enumerate cache misses with an $\mathbb{A}$-way associative cache using the strategy outlined above. We introduce two more pieces of notation to ease discussion. First, let $E_{0}$ and $E_{1}$ be the number of $\sigma_{i}$ with $i \geqslant \rho$ which are equal to 0 and 1 , respectively. Let $E=E_{0}+E_{1}$. Note that $E=\max \{2 m-\rho, 0\}$. Second, when referring to a variable that occurs in one of the equations Eq. (43)-Eq. (46) we will use $\bar{r}$ to denote the digits in the variable $r$ that occur in the equation taken $\bmod 2^{\rho}$ and $(p, q, r)_{2^{\rho}}$ to denote that all variables in the tuple should be taken $\bmod 2^{\rho}$; i.e., triple $(p, q, r)_{2^{\rho}}$ contains $\bar{p}, \bar{q}$, and $\bar{r}$. Note that $\bar{r}=r$ if $E=0$. Let us first enumerate $\mathbf{C}-\mathbf{A}$ collisions.

\subsection{Enumeration of $\mathbf{C}-\mathbf{A}$ collisions}

Step 1. Using the methods from Sections 2-4, determine NS, the number of triples $(i, j, u)_{2^{\rho}}$ which satisfy Eq. (43). Using ALGORITHM D1 from Lemma 5.1, 
determine ND, the number of triples $(i, j, u)_{2^{\rho}}$ such that $(i, j, u)_{2^{\rho}}$ and $(i, j, u-1)_{2^{\rho}}$ simultaneously satisfy Eq. (43).

Step 2. There are $(N S-N D) \cdot 2^{E_{0}+2 E_{1}}$ iteration steps $(i, k, j)$ at which there has been a single $\mathbf{C}-\mathbf{A}$ collision since the previous access of $C_{i, j}$. There are $N D \cdot 2^{E_{0}+2 E_{1}}$ iteration points $(i, k, j)$ at which there have been two $\mathbf{C}-\mathbf{A}$ collisions since the previous access of $C_{i, j}$.

\subsection{Enumeration of $\mathbf{C}-\mathrm{B}$ collisions}

This is the far more interesting case because elements of both $\mathrm{B}$ and $\mathrm{C}(C[i, j]$ and $B[k, j])$ are less "well behaved" than those $A[i, k]$ of array A. Thus, subsequently we show the approach in full length.

Step 1. Using the methods from Sections 2-4, determine NS, the number of triples $T=(i, j, z)_{2^{\rho}}$ having the property that there is a $\bar{y}$ such that $(i, j, z, y)_{2^{p}}$ satisfies Eq. (44). To each such triple $T$ we attach a multiplicity $m[T]$, this being the number of $\bar{y}$. Equation (44) almost completely determines $\bar{y}$; however, this multiplicity may arise if there is more than one choice of initial digits for $\bar{y}$, which give the same carry in $\mu_{2}+\Theta(z, y)$ from digits 0,1 to digit 2. Using ALGORITHM D2 from Lemma 5.2, determine ND, the number of triples $(i, j, k)_{2^{\rho}}$ such that there are simultaneous solutions $(i, j, k, u)_{2^{\rho}}$ and $(i, j, k-1, x)_{2^{\rho}}$ to Eq. (44). Also, using ALGORITHM D2, determine ND1, the number of triples $(i, j, k)_{2^{\rho}}$ such that there are simultaneous solutions $(i, j, k, u)_{2^{\rho}}$ and $(i, j, k-1, x)_{2^{\rho}}$ to Eq. (44) with $\bar{x} \leqslant j<\bar{u}$. Again, we will attach a multiplicity to each of these solutions.

Step 2. The next step differs significantly depending on whether $E>0$ or $E=0$, i.e, whether $2 m \leqslant \rho$ or $2 m>\rho$. We divide into those two cases, of which the latter is the more interesting.

Case 1: $E=0$

In this case, our enumeration is straightforward. There are ND1 iteration points $(i, k, j)$ in which there are two collisions of the forms $\gamma_{1}=(i, k, j, u)$ and $\gamma_{2}=(i, k-1, j, x)$. Each of $\gamma_{1}$ and $\gamma_{2}$ must be counted according to its multiplicity. There are $N S-2 \cdot N D 1$ iteration points where there has been a unique collision (which must be counted with multiplicity).

\section{Case 2: $E>0$}

In this case, the enumeration is more challenging. Consider a solution $T=$ $(i, z, j)_{2^{\rho}}$ counted in the number $N S$. It is enumerated because there exists a $\bar{y}$ such that $(i, j, z, y)_{2^{\rho}}$ is a solution to Eq. (44). Let $T_{2^{\rho}}=(i, z-1, j)_{2^{\rho}}$. Assume first that $T_{2^{\rho}}$ is not also a solution to Eq. (44). Let $(i, k, j)$ be any triple of numbers that extend $T$. Any extension of $\bar{y}$ will count a collision that occurs when $B_{k, j}$ is accessed at iteration step $(i, k, y)$ so long as $y \leqslant j$. Let $\phi(j)=\left\lfloor\frac{j}{2^{\rho}}\right\rfloor$ and let $\phi(y)=\left\lfloor\frac{y}{2^{\rho}}\right\rfloor$. If $\phi(y)<\phi(j)$, then $y<j$. If $\phi(y)>\phi(j)$ then $y>j$. If $\phi(y)=\phi(j)$, then $y \leqslant j$ iff $\bar{y} \leqslant \bar{j}$. So, $\phi(j)$ is an estimate for the number of collisions that is correct to within one. 
On the other hand, the extension of $y$ is arbitrary. So, for every solution $T=(i, z, j)_{2^{\rho}}$ to Eq. (44) counted by NS which is not counted by ND, and for every choice of $\phi \in\left\{0,1, \ldots, 2^{E_{1}}-1\right\}$ there are $2^{2 \cdot E_{0}}$ iteration steps $(i, k, j)$ for which the number of $\mathbf{C}-\mathbf{B}$ collisions is $\phi$ times the multiplicity of the triples, and this estimate is correct to within the multiplicity.

Assume now that $T_{2^{\rho}}$ is also a solution to Eq. (44). Such pairs $\left(T, T_{2^{\rho}}\right)$ are enumerated by ALGORITHM D2: their contribution to the analysis above must be subtracted out as a first step. In this case, every one of the $2^{\left(2 E_{0}+E_{1}\right)}$ extensions $(i, k, j)$ of $T=(i, z, j)_{2^{\rho}}$ is an iteration step at which there have been $m[T] \cdot 2^{E_{1}}$ collisions between the access of $C_{i, j}$ at iteration step $(i, k-1, j+\tau)$ and current access.

The reasoning is as follows. Let $(i, z, j, u)_{2^{\rho}}$ and $(i, z-1, j, x)_{2^{\rho}}$ be the simultaneous solutions to Eq. (48). There are $2^{\left(2 E_{0}+E_{1}\right)}$ extensions of $(i, z, j)_{2^{\rho}}$ to a triple $(i, k, j)$. Let $\phi \in B_{E_{1}}$. If $\phi \leqslant \phi(j)$ then we assign $\phi$ to be an extension of $\bar{u}$ to a $u \leqslant j$ so that there is a collision when $B_{k, u}$ is accessed at iteration step $(i, k, u)$. If $\phi>\phi(j)$ then we assign $\phi$ to be an extension of $s \bar{x}$ to an $x>j$ so that there is a collision when $B_{k-1, x}$ is accessed at iteration step $(i, k-1, x)$.

\subsection{Enumeration of $\mathbf{C}-\mathrm{C}$ collisions}

Let $N S$ be the number of solutions to Eq. (46) which we can compute using the methods in Sections 2-4. For every choice of solution $(i, j, y)_{2^{\rho}}$ to Eq. (46) there are $2^{E_{0}+E_{1}}$ ways to extend $\bar{l}, \bar{j}$ to $i, j \in B_{m}$. For each such pair of extensions there are $2^{m}$ ways to choose a $k$ to complete the determination of the iteration step $(i, k, j)$. Then every one of the $2^{E_{1}}$ possible extensions of $\bar{y}$ to $y \in B_{m}$ indexes a collision between $C_{i, y}$ and $C_{i, j}$ that occurs between the access of $C_{i, j}$ at iteration step $(i, k-1, j+\tau)$ and the access at iteration step $(i, k, j)$. If $y<j$ then the collision occurs at the iteration step $(i, k, y)$, whereas if $y>j$ then the collision occurs at iteration step $(i, k-1, y)$. There is one exception to this analysis. Clearly $\bar{y}=\bar{j}$ is a solution to Eq. (46) if and only if the choice of initial digits for $\bar{y}$ and $\bar{j}$ are identical. So, it is straightforward to compute $R$, the number of solutions to Eq. (46) in which $\bar{y}=\bar{j}$. The significance of these $R$ solutions, is that if $\bar{y}=\bar{j}$, then $y=j$ is a possible extension of $\bar{y}$ in which case, the collision we count above is not genuine.

To summarize, there are $N S \cdot 2^{E_{0}+E_{1}+m}$ iteration points where there have been C-C collisions. Of these, in $R \cdot 2^{E_{0}+E_{1}+m}$ cases there have been $2^{E_{1}}-1$ collisions and in $(N S-R) \cdot 2^{E_{0}+E_{1}+m}$ there have been $2^{E_{1}}$ collisions.

The following chart summarizes the analysis of $\mathbf{C}-\mathbf{A}, \mathbf{C}-\mathbf{B}$, and $\mathbf{C}-\mathbf{C}$ collisions above.

Type Number of iteration points

Number of collisions

$\begin{array}{llll}C-A & N S_{\text {equation (43) }} \cdot 2^{E_{0}+2 \cdot E_{1}} & 1 \text { or } 2 & \\ C-B_{1} & \left(N S_{\text {equations (44) }}-2 \cdot N S_{\text {equation }(48)}\right) \cdot 2^{2 \cdot E_{0}} \cdot\left(\# \phi<2^{E-1}\right) & \phi & z=k \\ & & 2^{E_{1}}-\phi & z=k-1 \\ C-B_{2} & N S_{\text {equation (48) }} \cdot 2^{2 \cdot E_{0}+E_{1}} & 2^{E_{1}} & \\ C-C & N S \cdot 2^{E_{0}+E_{1}+m} & 2^{E_{1}} \text { or }\left(2^{E_{1}}-1\right)\end{array}$


It remains to enumerate iteration points that fall into more than one of those categories. To understand the need for this, assume for example that $2^{E_{1}}<\mathbb{A}<$ $2^{2 \cdot E_{1}}$. Then no iteration point would exhibit more than $\mathbb{A}$ collisions of a single type $\mathbf{C}-\mathbf{A}, \mathbf{C}-\mathbf{B}$, or $\mathbf{C}-\mathbf{C}$. However, if there exists and iteration point that is simultaneously of type $\mathbf{C}-\mathbf{B}_{2}$ and $\mathbf{C}-\mathbf{C}$, then there would be at least $2^{2 \cdot E_{1}}>\mathbb{A}$ collisions between the access of $C_{i, j}$ at $(i, k-1, j+\tau)$ and the access at $(i, k, j)$. So there would be a C miss at $(i, k, j)$ with an $\mathbb{A}$-way associative cache.

The way we proceed is largely similar to what we have already shown in Section 4 for the direct mapped case. The lengthy analysis shown in Appendix A.3 yields a method to enumerate iteration points by number of $\mathbf{C}$ collisions. Using this method, we can determine $\phi(C, t)$, the number of iteration points $(i, k, j)$ for which there have been exactly $t$ collisions with $C_{i, j}$ between the prior access of $C_{i, j}$ and the access at $(i, k, j)$. Assuming an $\mathbb{A}$-way associative cache with a LRU protocol the number of $\mathbf{C}$ misses is $\sum_{t \geqslant A} \phi(C, t)$.

At this point we have indicated how to enumerate $\mathbf{A}$ misses and $\mathbf{C}$ misses in the case of an $\mathbb{A}$-way associative cache. It remains to enumerate $\mathbf{B}$ misses. Since the technical difficulties we encounter, as well as the ideas we use to overcome these difficulties, are similar to those seen in the enumeration of $\mathbf{C}$ misses we leave details to the reader.

The extension to first in first out (FIFO) replacement is straightforward. Here, the requirement that the accessed matrix elements are different is dropped in the definition of a collision.

\section{CONCLUSIONS}

This paper introduced a class of array layouts, interleavings, and efficient algorithms to exactly assess the number of cache misses caused by such layouts when used in the context of matrix multiplication. The layouts are described by bit-level address manipulations, and cache misses are counted by reasoning about the solutions to simple bit-level equations. Most importantly, we achieve a reduction in complexity from $\mathcal{O}\left(2^{m}+\rho\right)$ to $\mathcal{O}(\max (m, \rho))$ with respect to the naive algorithm by exploiting properties of carry propagation. Although there are various subcases in the analysis of cache misses, each case can be ultimately reduced to one of two combinatorial enumeration problems.

A particular strength of our techniques is that it explicitly handles cross interference between arrays, which is generally considered to be difficult to handle. Also, our model allows an elegant extension to a set-associative cache with LRU replacement strategy.

Our current work has several limitations. First, we have thus far provided an analysis only of matrix multiplication, and for $2^{m} \times 2^{m}$ matrices at that. It seems likely that the ideas can be generalized to handle other computations, but this remains to be demonstrated. Second, a number of special cases arise in dealing with the least significant bits of $\sigma$ that are truncated when converting a memory address to a block address. Our restriction to a cache block size of four elements required us to handle only two bits of $\sigma$, but the problem could be more acute for larger 
block sizes (e.g., in analyzing TLB behavior). Finally, our use of inclusion-exclusion poses the imminent danger of combinatorical explosion when the interaction of many arrays has to be calculated. However, as mentioned in Section 2.3, this case can be adequately approximated as many of these intersections are empty or sparse.

Our immediate future work will tackle the optimization problem of determining layout functions that minimize the number of cache misses for matrix multiplication. There are also related problems - such as counting compulsory misses, differentiating capacity and conflict misses, and identifying cache contents at the end of executing a loop nest-for which efficient algorithms remain to be found.

\section{APPENDIX A}

\section{Proofs and Details for Section 5}

\section{A.1. Proof of Lemma 5.1}

Proof. First, the algorithm will loop over the possible first two digits of $\Theta(i, j)$. As before in Section 3, we will let $\mu_{3}^{\prime}+\Theta^{\prime}\left(i^{\prime}, j^{\prime}\right)$ denote the part of $\mu_{3}+\Theta(i, j)$ in digits 2 to $(\rho-1)$ where the carry from digits 0,1 are incorporated into $\mu_{3}^{\prime}$.

Similarly, loop over the possible first two digits of $\Theta(i, z)$ (some of which might have already been fixed because $i$ is common to $\Theta(i, j)$ and $\Theta(i, z)$. When $\Theta(i, z+1)$ is computed from $\Theta(i, z)$ there will be some carry $\epsilon \in B_{2}$ from the part of $z+1$ that occurs in the first two digits of $\Theta(i, z+1)$ to the part of $z+1$ that occurs in the digits $2-(\rho-1)$ of $\Theta(i, z+1)$. Note that $\epsilon$ is determined by the first two digits of $\Theta(i, z)$ that we are looping over. Lastly, let $\gamma_{1}$ and $\gamma_{2}$ be the carry from digit one to digit two in $\mu_{1}+\Theta(i, z)$ and $\mu_{1}+\Theta(i, z+1)$ respectively. We use the prime notation from Section 4 to denote digits $2-(\rho-1)$. Combining all this we have

$$
\mu_{3}^{\prime}+\Theta^{\prime}\left(i^{\prime}, j^{\prime}\right)=\mu_{1}^{\prime}+\Theta^{\prime}\left(i^{\prime}, z^{\prime}\right)+\gamma_{1}=\mu_{1}^{\prime}+\Theta^{\prime}\left(i^{\prime}, z^{\prime}+\epsilon\right)+\gamma_{2} .
$$

The equalities in the equation above are modulo $2^{\rho-2}$ but still it is clear that the only possible ways in which they can be realized are if

1. $\gamma_{1}=\gamma_{2}=\epsilon=0$

or

2. $\gamma_{1}=\epsilon=1, \gamma_{2}=0$ and $\sigma_{2}=1$.

So the algorithm proceeds in the following way. It loops over possible first two digits of $\Theta(i, j)$ and $\Theta(i, z)$. For each such choice, the algorithm computes $\gamma_{1}, \gamma_{2}$ and $\epsilon$. If neither 1 nor 2 above is satisfied, then there is no contribution to the total count and the algorithm proceeds to the next step in the loop. If either 1 and 2 above is satisfied, then the algorithm computes the number of solutions to

$$
\mu_{3}^{\prime}+\Theta^{\prime}\left(i^{\prime}, j^{\prime}\right)=\mu_{1}^{\prime}+\Theta^{\prime}\left(i^{\prime}, z^{\prime}\right)
$$

using the AC ALGORITHM and adds that number to the total. 


\section{A.2. Proof of Lemma 5.2}

Proof. We will need some terminology and notation to explain this algorithm. Let I be the number of 0 's in the set $\left\{\sigma_{0}, \sigma_{1}\right\}$. The initial digits of either $k$ or $k-1$ will refer to the first $I$, i.e., those that appear in the first two digits of $\Theta(k, v)$ and $\Theta(k-1, x)$. Let $v$ be the minimal index greater than 1 with $\sigma_{v}=0$. So we have $\sigma_{2}=\sigma_{3}=\cdots=\sigma_{v-1}=1$.

The first step in this algorithm is to loop over choices for the initial digits of $k-1$ (which will also determine the initial digits of $k$. Let $\tau \cdot 2^{I}$ be the carry when 1 is added to the initial digits of $k-1$. Note that $\tau$ is carried to the $v$ th binary digit when $\Theta(k, v)$ is computed from $\Theta(k-1, v)$.

The next step is to loop over possible carries $\epsilon_{0}, \epsilon_{1}$ and $\epsilon_{2}$ from the zero and first binary digits to the second binary digit in $\mu_{3}+\Theta(i, j), \mu_{2}+\Theta(k, v)$ and $\mu_{2}+\Theta(k-1, x)$, respectively. We compute the number $N_{0} N_{1} N_{2}$, where $N_{0}$ is the number of choices for the zero and first binary digits of $\mu_{3}+\Theta(i, j)$, which will result in a carry of $\epsilon_{0}$, where $N_{1}$ is the number of choices of initial digits of $v$ and $x$, respectively, which will result in carries of $\epsilon_{1}$ and $\epsilon_{2}$, respectively (given the choices we have already made for initial digits in $k-1$ and $k$ ).

With this notation and the prime notation we can express Eq. (48) as

$$
\mu_{3}^{\prime}+\Theta^{\prime}\left(i^{\prime}, j^{\prime}\right)+\epsilon_{0}=\mu_{2}^{\prime}+\Theta^{\prime}\left(k^{\prime}, v^{\prime}\right)+\epsilon_{1}=\mu_{2}^{\prime}+\Theta^{\prime}\left((k-1)^{\prime}, x^{\prime}\right)+\epsilon_{2} .
$$

So,

$$
\Theta^{\prime}\left((k-1)^{\prime}, v^{\prime}\right)+\epsilon_{1}+\tau \cdot 2^{v-2}=\Theta^{\prime}\left((k-1)^{\prime}, x^{\prime}\right)+\epsilon_{2} .
$$

Rewriting Eq. (52) we obtain,

$$
\left(\epsilon_{1}-\epsilon_{2}\right)+\tau \cdot 2^{v-2}=\Theta^{\prime}\left((k-1)^{\prime}, x^{\prime}\right)-\Theta^{\prime}\left((k-1)^{\prime}, v^{\prime}\right)
$$

Let $\hat{v}$ and $\hat{x}$ denote $v^{\prime}$ and $x^{\prime}$ taken $\bmod 2^{v-2}$. We note that the right-hand side of Eq. (53) is equal to

$$
\hat{x}-\hat{v}+G L O B
$$

where $G L O B$ is a multiple of $2^{v-1}$. Since the left-hand side of Eq. (53) is strictly less than $2^{v-1}$, we deduce that $G L O B=0$ and so

$$
\epsilon_{1}+\epsilon_{2}+\tau \cdot 2^{v-2}=\hat{x}-\hat{v}
$$

Case 1. $\tau=0$.

In this case, Eq. (54) becomes

$$
\epsilon_{1}+\hat{v}=\epsilon_{2}+\hat{x}
$$

Also, $\epsilon_{1}+\hat{v}=\epsilon_{2}+\hat{x}$ is completely determined by the equality equation (51). So there is exactly one choice of $\hat{v}$ and $\hat{x}$ in this case for every $i, j$. So in this case there 
are $\min \left\{2^{\rho-2}, 2^{2 m-2}\right\}$ choices for $i, j$. For each such choice there is exactly one choice of $k$. For this triple $(i, k, j)$ there are $N_{0} \cdot N_{1} \cdot N_{2}$ choices of $B_{w, z}$ that collide with $C_{i, j}$ between its access at iteration steps $(i, k-1, j+\tau)$ and $(i, k, j)$.

It remains to determine the number of these collisions satisfying $v \leqslant j<x$. The reader will note that

$$
\epsilon_{1}+v^{\prime}=\epsilon_{2}+x^{\prime}
$$

so there are no such $j$ unless $\epsilon_{1}=1, \epsilon_{2}=0$ and in this case any $j$ with $v \leqslant j<x$ must satisfy

$$
v^{\prime}=j^{\prime}<x^{\prime}=v^{\prime}+1 .
$$

The first thing to check is whether the choices of initial digits for $j, x, v$ would mean that $j^{\prime}, x^{\prime}, v^{\prime}$ which satisfy Eq. (56) would give $j, x, v$ with $v \leqslant j<x$. If not, then there are no such $j$. If so, we enumerate $j^{\prime}, x^{\prime}, v^{\prime}$ by enumerating solutions to

$$
\mu_{3}^{\prime}+\Theta^{\prime}\left(i^{\prime}, j^{\prime}\right)=\mu_{2}^{\prime}+\Theta^{\prime}\left(k^{\prime}, j^{\prime}\right)
$$

using the methods developed in Section 2.

Case 2. $\tau=1$.

In this case, Eq. (54) is equivalent to

$$
\hat{v}+\epsilon_{1}=\hat{x}+\epsilon_{2}+2^{v} .
$$

Recalling that $\hat{v}, \hat{x} \in B_{v}$, we see that Eq. (57) can have a solution only if $\hat{x}=0$, $\epsilon_{2}=0, \epsilon_{1}=1$ and $\hat{v}=2^{v}-1$. In order for these choices of $\hat{x}, \hat{v}, \epsilon_{1}$ and $\epsilon_{2}$ to satisfy Eq. (51) we must have that $\mu_{3}^{\prime}+j^{\prime}+\epsilon_{0}$ agrees with $\mu_{2}^{\prime}$ in digits $0-(v-3)$. This implies that digits $0-(\mu-3)$ in $j^{\prime}$ are determined by $\mu_{3}, \epsilon_{0}$ and $\mu_{2}$. So there are $\min \left\{2^{\rho-v}, 2^{2 m-v}\right\}$ choices for such $i, j$. Each determines a unique $k$ and $N_{0} \cdot N_{1} \cdot N_{2}$ pairs $w, z$ such that $B_{w, z}$ collides with $C_{i, j}$ between the access of $C_{i, j}$ at iteration steps $(i, k-1, j+\tau)$ and $(i, k, j)$.

Finally, we need to determine the number of these solutions which satisfy $v \leqslant j<x$. However, in this case, $v>x$ and so there are no such $j$.

This completes the proof of Lemma 5.2 and the construction of ALGORITHM D2.

\section{A.3. Enumeration of Iteration Points that Exhibit Simultaneous Collisions}

We show the case of the enumeration of iteration points that exhibit simultaneous $\mathbf{C}-\mathbf{A}$ and $\mathbf{C}-\mathbf{B}$ collisions and thus seek to enumerate iteration points $(i, k, j)$ such that there have been both $\mathbf{C}-\mathbf{A}$ and $\mathbf{C}-\mathbf{B}$ collisions between $(i, k-1, j+\tau)$ and $(i, k, j)$. We begin by enumeration simultaneous solutions to Eqs. (43) and (44) but with the added factor that either $u=z, u+1=z$ or $u=z+1$. We split our analysis into those three cases. 
We will consider only the case where $u=z$ here-the other cases can be handled via similar methods. In the case $u=z$, a simultaneous solution to Eq. (43) and Eq. (44) must satisfy

$$
\left\lfloor\frac{\mu_{3}+\Theta(i, j)_{2^{\rho}}}{4}\right\rfloor=\left\lfloor\frac{\mu_{1}+\Theta(i, z)_{2^{\rho}}}{4}\right\rfloor=\left\lfloor\frac{\mu_{2}+\Theta(z, y)_{2^{\rho}}}{4}\right\rfloor .
$$

We enumerate solutions to Eq. (58) by first counting solutions $\bar{j}, \bar{z}$ to the left-most equality using the AB-Algorithm, but keeping-the choice of $\bar{l}$ open for the moment. For each such solution $\bar{j}, \bar{z}$, there is one and only one choice of $\bar{l}$ and $\bar{y}$ that satisfies the second equality.

If $E=0$, then $i, j, z$, and $y$ are determined at this point. Also, $k$ is determined, as $k=z$ if $y \leqslant j$ and $k=z+1$ if $y>j$. For this iteration point $(i, k, j)$, the number of collisions will be 2, 3 or 4 . The normal case will be 2 but 3 or 4 may result if there are two $\mathbf{C}-\mathbf{A}$ collisions or two $\mathbf{C}-\mathbf{B}$ collisions (or both). We will discuss these cases below.

If $E>0$ then we must extend $\bar{l}, \bar{j}, \bar{z}$ and $\bar{y}$ to get $i, j, z$ and $y$. The consideration on extensions is identical to those above. In particular, $k$ will be either $z$ or $z+1$ depending on how the extension of $y$ compares to the extension on $j$. In cases where $\bar{z}$ and $\overline{z+1}$ are not both solutions to Eq. (44) then the number of collisions will be $1+\phi(j)$ or $2+\phi(j)$ for $k=z$ depending on whether there are multiple $\mathbf{C}-\mathbf{A}$ collisions (which is discussed below). The number of collisions will be $1+2^{E_{1}}-\phi(j)$ or $2+2^{E_{1}}-\phi(j)$ for $k=z+1$ depending on whether there are multiple $\mathbf{C}-\mathbf{A}$ collisions. However, if $\bar{z}$ and $\overline{z+1}$ are both solutions to Eq. (44) then the number of collisions will be either $1+2^{E_{1}}$ or $2+2^{E_{1}}$ depending on whether there are multiple $\mathbf{C}-\mathbf{A}$ collisions.

So we will need to enumerate simultaneous solutions to Eqs. (47) and (44), Eqs. (43) and (48), and Eqs. (47) and (48). These enumeration problems can be solved using the tools we have already developed and applied to counting solutions to Eqs. (43) and (44). So, we will omit many of the details in our account of how to proceed.

To enumerate simultaneous solutions to Eqs. (47) and (44), we begin by counting solutions to

$$
\left\lfloor\frac{\mu_{3}+\Theta(i, j)_{2^{\rho}}}{4}\right\rfloor=\left\lfloor\frac{\mu_{1}+\Theta(i, z)_{2^{\rho}}}{4}\right\rfloor=\left\lfloor\frac{\mu_{1}+\Theta\left(i, z+1_{2^{\rho}}\right)}{4}\right\rfloor=\left\lfloor\frac{\mu_{2}+\Theta(z, y)_{2^{\rho}}}{4}\right\rfloor .
$$

To count solutions to this equation, use Lemma 5.1 to enumerate solutions $\bar{j}, \bar{z}$ to the first two equalities leaving $\bar{l}$ undecided. With $\bar{J}, \bar{z}$ fixed there are unique choices for $\bar{l}$ and $\bar{y}$ which satisfy the third equality.

Each choice of $\bar{j}, \bar{z}, \bar{l}$, and $\bar{y}$ which satisfy Eq. (59) must now be extended. First, note that there are $2^{E_{0}+2 \cdot E_{1}}$ ways to extend each solution $\bar{j}, \bar{z}, \bar{l}$. Observe that the extension of $z$ must be $k-1$ because of the form of Eq. (59). For each such extension, there are $2+Y$ collisions of types $\mathbf{C}-\mathbf{A}$ and $\mathbf{C}-\mathbf{B}$ at iteration point $(i, k, j)$, 
where $Y$ is the number of extensions of $\bar{y}$. Since $z=k-1$, the $Y$ is the number of extensions of $\bar{y}$ is $2^{E_{1}}-\phi(j)$, where $\phi(j)$ is the extension of $j$.

As noted in the previous paragraph, the form of Eq. (59) imply that $z=k-1$. So we must also solve a second set of equations which differ from Eq. (59) only in that the last $\bar{z}$ is replaced by $\overline{z+1}$. The enumeration of solutions to this set of equations follows the lines above with the only major difference being that there are $\phi(j)$ extensions possible for $\bar{y}$. This impacts the number of collisions that have occurred at iteration step $(i, k, j)$.

To enumerate simultaneous solutions to Eqs. (43) and (48), we must count solutions to the system of equations

$$
\left\lfloor\frac{\mu_{3}+\Theta(i, j)_{2^{\rho}}}{4}\right\rfloor=\left\lfloor\frac{\mu_{1}+\Theta(i, z)_{2^{\rho}}}{4}\right\rfloor=\left\lfloor\frac{\mu_{1}+\Theta(z, y)_{2^{\rho}}}{4}\right\rfloor=\left\lfloor\frac{\mu_{2}+\Theta(z+1, x)_{2^{\rho}}}{4}\right\rfloor .
$$

To enumeration of solutions to Eq. (60), we use the AB Algorithm to count solutions to the second equality. The reasoning that went into the proof of Lemma 5.2 give (in each of two cases), the relationship between $\bar{y}$ and $\bar{x}$. The one complication that arises is in the case (from Lemma 5.2) where $\tau=1$. In this case, the first $v$ digits of $y^{\prime}$ are determined and so the first $v$ digits of $\bar{z}^{\prime}$ will also be determined. This gives a partial determination of $\bar{z}$ which must be factored into the AB Algorithm as was done in Section $=3$.

Each solution of Eq. (60) must be extended. The number of extensions is straightforward to count in this case, since there are $2^{E_{1}}$ extensions of the pair $\bar{y}, \bar{x}$. To complete this analysis, we must enumerate simultaneous solutions to Eqs. (47) and (48). We begin by enumerating solutions to

$$
\begin{aligned}
\left\lfloor\frac{\mu_{3}++\Theta(i, j)_{2^{\rho}}}{4}\right\rfloor & =\left\lfloor\frac{\mu_{1}+\Theta(i, z)_{2^{\rho}}}{4}\right\rfloor=\left\lfloor\frac{\mu_{1}+\Theta(i, z+1)_{2^{\rho}}}{4}\right\rfloor \\
& =\left\lfloor\frac{\mu_{1}+\Theta(z, y)_{2^{\rho}}}{4}\right\rfloor=\left\lfloor\frac{\mu_{2}+\Theta(z+1, x)_{2^{\rho}}}{4}\right\rfloor .
\end{aligned}
$$

To characterize solutions to Eq. (61), begin with the first two equalities. Following the reasoning in the proof of Lemma 5.1, we can eliminate some choices for initial digits of $\bar{j}, \bar{z}, \bar{l}, \bar{x}$, and $\bar{y}$. For those that are not eliminated, solutions of Eq. (61) are equivalent to solutions of Eq. (60) and so we can use the methods developed above to enumerate those solutions. As in other cases, once solutions to Eq. (61) are enumerated, they must be extended to give simultaneous solutions to Eqs. (47) and (48). These extensions will then give the number of iteration points. At each there will be $2+2^{E_{1}}$ collisions between the access of $C_{i, j}$ at that iteration step and the most recent previous access.

This completes the enumeration of iteration steps where there have been both $\mathbf{C}-\mathbf{A}$ and $\mathbf{C}-\mathbf{B}$ collisions. The next step is to enumerate iteration points where there have been both $\mathbf{C}-\mathbf{A}$ and $\mathbf{C}-\mathbf{C}$ collisions, iteration points where there have been both $\mathbf{C}-\mathbf{B}$ and $\mathbf{C}-\mathbf{C}$ collisions, and iteration points where there have been all three 
of $\mathbf{C}-\mathbf{A}, \mathbf{C}-\mathbf{B}$, and $\mathbf{C}-\mathbf{C}$ collisions. To shorten this exposition, we will only indicate where the modifications of the previous analyses come in.

To enumerate cases where there have been both $\mathbf{C}-\mathbf{A}$ and $\mathbf{C}-\mathbf{C}$ collisions is straightforward. Enumerate solutions $\bar{j}, \bar{u}$ of to Eq. (43) including the number for which both $\bar{\jmath}, \bar{u}$ and $\bar{j}, \overline{u-1}$ are solutions. For each of these, there is at most one $\bar{y}$ such that $\bar{y}, \bar{y}$ satisfy Eq. (46). There will exist an $\bar{y}$ unless the carries from the initial two digits of the two sides of Eq. (46) are different. In this case you may have to eliminate one possible solutions to Eq. (43). This determination can be made by an $O(\rho)$ examination of $\mu_{1}$ and $\mu_{3}$. Once $\bar{j}, \bar{u}$ and $\bar{y}$ have been determined, we can choose $i$ arbitrarily and we can arbitrarily extend $\bar{y}, \bar{u}$ and $\bar{y}$ to $j, u$ and $y$. Different extensions of $i, j$ and $u$ lead to different iteration points. However, for fixed $i, j$ and $u$, different extensions of $\bar{y}$ correspond to multiple collisions.

To enumerate cases where there have been both $\mathbf{C}-\mathbf{B}$ and $\mathbf{C}-\mathbf{C}$ collisions is likewise straightforward. First enumerate solutions to Eq. (46). For every solution $\bar{l}, \bar{\jmath}, \bar{v}$, there is a uniquely determined solution to $\bar{z}, \bar{y}$ up to multiplicity $m[T]$ that might arise from differing initial digits in $\Theta(z, y)$. As before, we must determine whether different choices of initial digits might lead to both $\bar{z}$ and $\overline{z+1}$ being solutions. This is straightforward. Each solution $\bar{l}, \bar{j}, \bar{v}, \bar{z}, \bar{y}$ just enumerated must be extended. The issues related to extensions are identical to the issues that arose in the enumeration of $\mathbf{C}-\mathbf{B}$ collisions. We leave details to the reader.

Finally, we need to enumerate cases where there have been $\mathbf{C}-\mathbf{A}, \mathbf{C}-\mathbf{B}$, and $\mathbf{C}-\mathbf{C}$ collisions. We first enumerate iteration points where there have been $\mathbf{C}-\mathbf{A}$ and $\mathbf{C}-\mathbf{C}$ collisions as above. For every such solution, we can uniquely solve for $\bar{z}, \bar{y}$, uniquely up to choice of initial digits. As usual, consideration must be given to whether there $\bar{u}$ and $\overline{u-1}$ are both solutions to Eq. (43) and to whether $\bar{z}$ and $\overline{z+1}$ are both solutions to Eq. (44). There are no novel issues that arise around extensions and so again we leave details to the reader.

\section{REFERENCES}

1. S. Chatterjee, V. V. Jain, A. R. Lebeck, S. Mundhra, and M. Thottethodi, Nonlinear array layouts for hierarchical memory systems, in "Proceedings of the 1999 ACM International Conference on Supercomputing," pp. 444-453, Rhodes, Greece, June 1999.

2. S. Chatterjee, A. R. Lebeck, P. K. Patnala, and M. Thottethodi, Recursive array layouts and fast parallel matrix multiplication, in "Proceedings of the Eleventh Annual ACM Symposium on Parallel Algorithms and Architectures,” pp. 222-231, Saint-Malo, France, June 1999.

3. P. Feautrier, Dataflow analysis of array and scalar references, Internat. J. Parallel Programming 20(1) (1991), 23-54.

4. J. D. Frens and D. S. Wise, Auto-blocking matrix-multiplication or tracking BLAS3 performance with source code, in "Proceedings of the Sixth ACM SIGPLAN symposium on Principles and Practice of Parallel Programming," pp. 206-216, Las Vegas, NV, June 1997.

5. S. Ghosh, M. Martonosi, and S. Malik, Cache miss equations: A compiler framework for analyzing and tuning memory behavior, ACM Trans. Prog. Lang. Systems 21(4) (1999), 703-746.

6. J. L. Hennessy and D. A. Patterson, "Computer Architecture: A Quantitative Approach,” 2nd ed., Morgan Kaufmann, San Mateo, CA, 1996.

7. M. D. Hill and A. J. Smith, Evaluating associativity in CPU caches, IEEE Trans. Comput. C 38(12) (1989), 1612-1630. 
8. M. S. Lam, E. E. Rothberg, and M. E. Wolf, the cache performance and optimizations of blocked algorithms, in "Proceedings of the Fourth International Conference on Architectural Support for Programming Languages and Operating Systems," pp. 63-74, Apr. 1991.

9. W. Pugh, The Omega test: A fast practical integer programming algorithm for dependence analysis, Comm. Assoc. Comput. Mach. (Aug. 1992), 102-114. 